\title{
Feasibility of Catalpol Intranasal Administration and Its Protective Effect on Acute Cerebral Ischemia in Rats via Anti-Oxidative and Anti-Apoptotic Mechanisms
}

\author{
Jinghui Wang' \\ Yuhua Zhang ${ }^{2}$ \\ Meifeng Zhang' \\ Si Sun' \\ Yang Zhong' \\ Lei Han' \\ Yitong $\mathrm{Xu}^{\prime}$ \\ Dong Wan (1D ${ }^{3}$ \\ Junhui Zhang ${ }^{4}$ \\ Huifeng Zhu (D)' \\ 'College of Pharmaceutical Sciences \& \\ College of Chinese Medicine, Southwest \\ University, Chongqing, 4007I5, People's \\ Republic of China; ${ }^{2}$ Central Hospital of \\ Enshi Tujia and Miao Autonomous \\ Prefecture, Enshi, Hubei, 445000, \\ People's Republic of China; ${ }^{3}$ Department \\ of Emergency and Critical Care Medicine, \\ the First Affiliated Hospital of Chongqing \\ Medical University, Chongqing, 400016, \\ People's Republic of China; ${ }^{4}$ Health \\ Management Center, The First Affiliated \\ Hospital of Chongqing Medical University, \\ Chongqing, 400016, People's Republic of \\ China
}

Correspondence: Huifeng Zhu

College of Pharmaceutical Sciences and Traditional Chinese Medicine, Southwest University, Chongqing, 4007I5, People's Republic of China

Email zhfbsci@I26.com

Junhui Zhang

Health Management Center, The First Affiliated Hospital of Chongqing Medical University, Chongqing, 4000 I6, People's Republic of China

Email2275610878@qq.com
Purpose: Catalpol is the main active component of Rehmannia glutinosa, which has a variety of pharmacological activities, including anti-inflammatory and anti-oxidative effects. This study investigates the feasibility of catalpol intranasal administration and its protective effect on acute cerebral ischemia in rats via anti-oxidative and anti-apoptotic mechanisms.

Patients and Methods: This study investigates the method of catalpol intranasal administration to evaluate the nasal mucosal toxicity, brain targeting and pharmacokinetics of catalpol. The protective effect of catalpol of intranasal administration on stroke-induced brain injury in rats and its mechanisms on oxidative stress pathway $\mathrm{Nrf} 2 / \mathrm{HO}-1$ and apoptosis were also investigated using middle cerebral artery occlusion (MCAO).

Results: The results showed that catalpol intranasal administration was safe and feasible with no hemolysis, no bad effect on the maxillary ciliary movement of bullfrog. After intranasal administration, the brain targeting index (DTI) of catalpol was greater than 1, which indicated that catalpol had good brain targeting after intranasal administration. The bioavailability of catalpol administered intranasally was higher than that of in plasma. In MACO model, catalpol intranasal administration could significantly reduce cerebral infarction volume, neurological dysfunction and brain edema. In addition, catalpol intranasal administration can also reduce the brain cell's occurrence of apoptosis, promote the expression of Bcl-2 protein and inhibit the expression of Bax protein, reduce oxidative stress damage via up-regulating expression of $\mathrm{Nrf} 2$ and $\mathrm{HO}-1$, increasing the activities of SOD and decreasing the activities of MDA.

Conclusion: Collectively, catalpol intranasal administration has good safety, stability and brain targeting. It can effectively protect the brain injury of the rat model of acute cerebral ischemia and provide the possibility of drug administration in the acute stage of cerebral ischemia, especially before entering the hospital.

Keywords: catalpol, cerebral ischemia, intranasal administration, oxidative stress, apoptosis, neuroprotection

\section{Introduction}

Stroke is the most common neurological disease with high morbidity, mortality and disability. ${ }^{1}$ Stroke can be divided into ischemic and hemorrhagic, of which $85 \%$ is ischemic stroke. ${ }^{2}$ Early use of drug in acute phase is the key to the treatment of ischemic stroke. ${ }^{3}$ Cerebral ischemia patients in a short period of time are very likely to appear on one side hemiplegia, aphasia, more serious coma or death. ${ }^{4}$ 


\section{Graphical Abstract}

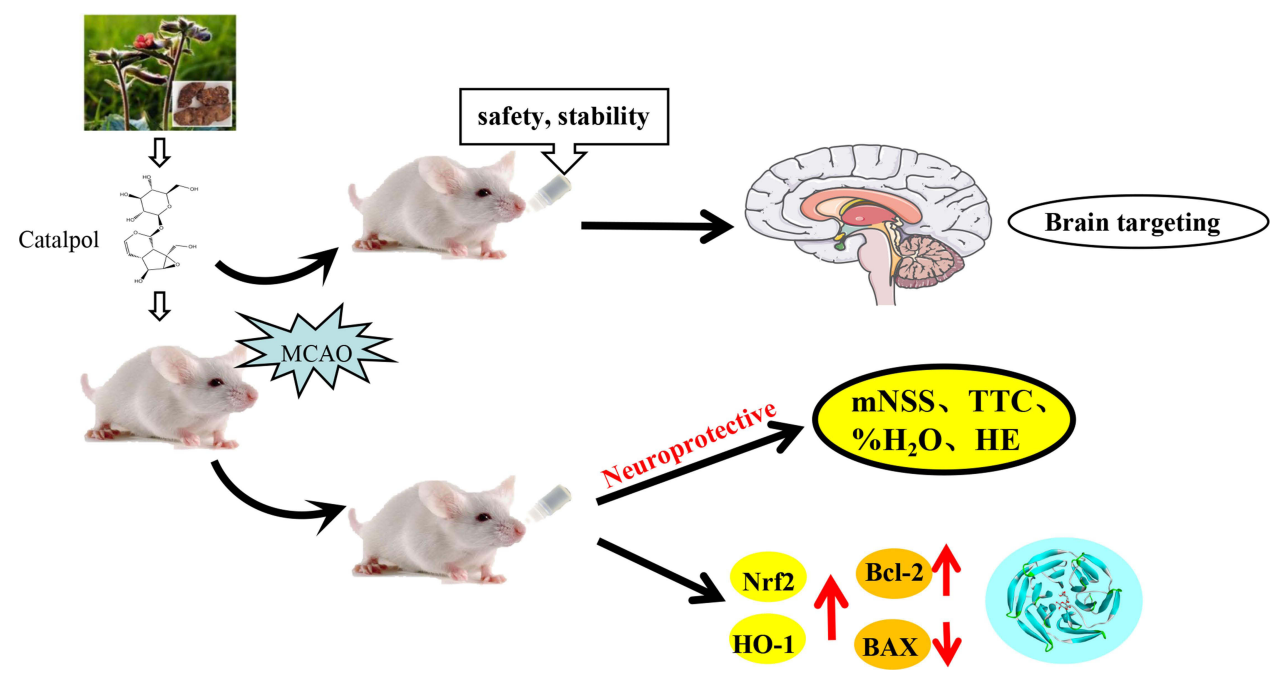

Because the onset of cerebral ischemia is urgent, the attack is not predictable, and the disability rate and mortality rate are high, rapid control is the key to treatment. After cerebral ischemia, there will be disturbance of consciousness and difficulty in swallowing. The traditional way of administration such as via intravenous injection or to be taken orally have disadvantage. For example, intravenous injections need to be done in hospital; Drug taken orally is dangerous because these stroke patients are often in the state of disturbance of consciousness. So the intranasal administration has the advantages of convenient operation, rapid absorption and quick effect, and can quickly transmit the drug to the brain to facilitate first aid and self-rescue.

Cerebral ischemia injury is a complex pathophysiological process. ${ }^{5}$ Cerebral blood flow disruption causes brain tissue ischemia and hypoxia, oxidative stress injury, inflammatory response and apoptosis, which occur at different time points and are closely related. ${ }^{6}$ The accumulation of various damage factors eventually lead to irreversible damage of brain tissue. ${ }^{7}$ Oxidative stress is closely related to neuronal death after cerebral ischemia. ${ }^{8}$ The activation of antioxidant system in the body plays an important role in the survival of nerve cells after cerebral ischemia. ${ }^{9} \mathrm{Nrf} 2$ is a key factor in oxidative stress response, which can regulate the expression of antioxidant proteins and the expression of HO-1 factor. Studies have shown that Nrf2/HO-1 pathway plays an important antioxidant role. ${ }^{10}$ The key elements of the apoptotic pathway include the changes in the expression of the pro-apoptotic protein Bax and the apoptotic protein Bcl-2. ${ }^{11}$

Catalpol is one of the iridoid glycoside isolated from Rehmannia glutinosa. ${ }^{12}$ Catalpol has a variety of pharmacological activities, including anti-inflammatory and antioxidative effects. ${ }^{13}$ Catalpol can treat cerebral ischemia, diabetic encephalopathy, hyperlipidemia, Parkinson's disease, $^{14}$ dementia and other neurological diseases. ${ }^{15}$ Catalpol has anti-inflammatory and antioxidant effects. ${ }^{16}$ In vitro, it attenuated $\mathrm{H}_{2} \mathrm{O}_{2}$-induced apoptosis of $\mathrm{PC} 12$ cells. ${ }^{17}$ In vivo, the antioxidant properties of catalpol has been well studied in gerbils. ${ }^{18}$

In this study, catalpol intranasal administration was used to introduce catalpol into the brain without injury to evaluate the toxicity of catalpol nasal mucosa. Evaluated the therapeutic effect of catalpol intranasal administration with middle cerebral artery occlusion (MCAO) ischemic stroke model. We determined the effect of catalpol on infarct volume and neurological dysfunction caused by MCAO in rats. We further study the cellular stress response pathway $\mathrm{Nrf} / \mathrm{HO}-1$ and apoptosis pathway $\mathrm{Bcl}-$ 2 in the potential role of catalpol in the treatment of cerebral ischemic injury.

\section{Materials and Methods}

\section{Chemicals and Antibodies}

Catalpol (purity $>98 \%$ ) was purchased from Liu Bo Bai Niao Biological Technology Co., Ltd. (Shi Jiazhuang, 
China). Catalpol (Standard products) was purchased from National Institute for the Control of Pharmaceutical and Biological Products (Chengdu, China). The chemical structures of catalpol and aucubin are given in HPLC grade of methanol and acetonitrile was purchased from Shanghai Titan chem Co., Ltd. (Shanghai, China). Ultrapure water was produced using a Millipore water purification system (Millipore, Molsheim, France). The antibodies against $\mathrm{Nrf} 2$ and $\mathrm{HO}-1$ were obtained from Santa Cruz Biotechnology (CA, USA). Antibody against Bcl-2, BAX and $\beta$-actin was obtained from Proteintech (Wuhan, China).

\section{Animals}

All experimental procedures involving animals were approved by the Animal Ethics Committee at Southwest University. All experimental procedures conformed to China's Guidelines for the Care and use of laboratory animals. Male Sprague-Dawley (220-250 g) rats at youth stage were provided by the Experimental Animal Center, Chongqing Medicine University. All rats were housed in controlled temperature $\left(22 \pm 2^{\circ} \mathrm{C}\right)$ and lighting $(12 \mathrm{~h}$ light/ dark cycle) with free access to standard food and drinking water.

\section{Hemolysis Test}

Rabbit blood $(4 \mathrm{~mL})$ was anticoagulated with $0.1 \mathrm{~mL}$ of heparin sodium and then washed with $2 \mathrm{~mL}$ of normal saline, followed by centrifugation at $1800 \mathrm{r} / \mathrm{min}$ for 10 min. After supernate was discarded, the solution was washed three times. The remaining erythrocytes were prepared into $2 \%$ normal saline solution. Then, $10 \mathrm{mg} / \mathrm{mL}$ catalpol aqueous solutions were prepared.

Then, $2.5 \mathrm{~mL}$ of erythrocyte suspension was each added to 15 dry test tubes, followed successively by addition of distilled water, normal saline, and $2.5 \mathrm{~mL}$ of 1,5 , $10 \mathrm{mg} / \mathrm{mL}$ catalpol solutions. The tubes were incubated at $37^{\circ} \mathrm{C}$ for $2 \mathrm{~h}$.

\section{Ciliotoxicity Test}

When bullfrogs were fixed in dorsal position on a frog board, $3 \mathrm{~mm} * 3 \mathrm{~mm}$ of palatine mucosa was separated by scissors and tweezers. After blood was washed off by normal saline, the palatine mucosa was spread with the ciliated surface upwards on a glass slide, and added onto surface with deoxysodium cholate, normal saline $10 \mathrm{mg} /$ $\mathrm{mL}$ catalpol solutions for observation of persistent vibration duration (PVD) of cilium. Percentage of persistent vibration (PPV) is calculated as PVD of each group divided by PVD of normal saline. A higher PPV indicates lower toxicity.

\section{Histopathology for Nasal Mucosal Toxicity and Tolerability}

The 48 healthy SD rats $(200 \pm 20 \mathrm{~g})$ were randomly divided into three groups: control (normal saline), deoxycholate solution and catalpol group, with 12 rats in each group. After the rats were anesthetized, they were kept supine, and the neck was slightly raised to make it $45^{\circ}$. The dose was $10 \mathrm{mg} / \mathrm{kg}$ alternate administration on both sides of the nasal cavity. After the administration on one nostril, waiting for it to be absorbed and then given to the other side. Each administration $10 \mu \mathrm{L}$ with an interval of 2 minutes each time. The whole administration process lasts for 30 minutes. After intranasal for 7 days, the drug was stopped for one week. Nasal mucosa was collected and fixed in $4 \%$ paraformaldehyde on day 1 , day 4 , day 7 and day 7 after drug withdrawal. Tissue blocks were prepared using paraffin beeswax and dried in a hot-air oven, then sliced using a microtome at $4 \mu \mathrm{m}$. The obtained slices were then deparaffinized and stained using hematoxylin and eosin stains and examined using a light microscope agated tissues.

\section{HPLC Analysis}

Catalpol was performed on a Agilent HPLC system (Agilent 1200 series with a G1322A vacuum degasser, G1311 AQuat pump, G1329 Autosampler, G1316A thermostatted column compartment, G1365D Multi-Wavelength Detector, and G1328B Man. Inj, in conjunction with Agilent ZORBAX bonus-rp C18 column $(250 \mathrm{~mm} \times$ $4.6 \mathrm{~mm}, 5 \mu \mathrm{m}$, Agilent Technologies, American). The mobile phase was a mixture of Acetonitrile and water (0.5:99.5, $\mathrm{v} / \mathrm{v}$ ) with a flow rate of $1 \mathrm{~mL} / \mathrm{min}$. The auto-sampler was maintained at $25^{\circ} \mathrm{C}$, and the injection volume was held constant at $10 \mu \mathrm{L}$.

\section{Brain Targeting Evaluation of Catalpol Intranasal}

Sample collection: 30 male SD rats $(220 \pm 10 \mathrm{~g})$ were selected. They were randomly divided into intravenous group (i.v.) and intranasal group (i.n.), with 15 rats in each group. The dose is $10 \mathrm{mg} / \mathrm{kg}$. The rats were sacrificed $15,30,60,120$ and 180min after administration, respectively, and blood was collected from the abdominal aorta. Blood samples were placed for $30 \mathrm{~min}$ and centrifuged at 
$3000 \mathrm{r} / \mathrm{min}$ for $10 \mathrm{~min}$. Plasma was collected and stored at $-80^{\circ} \mathrm{C}$. The brain tissue of rats was completely removed, and parts of olfactorius bulbus, hippocampus, medulla oblongata, the cerebellum and cortex were quickly separated and stored at $-80^{\circ} \mathrm{C}$.

We took $0.5 \mathrm{~mL}$ of plasma sample or $0.1 \mathrm{~g}$ of brain tissue, $50 \mathrm{uL}$ of Asparagine solution was added and mixed. Then added $2.5 \mathrm{~mL}$ of acetonitrile, precipitated protein was mixed, centrifuged at $3500 \mathrm{r} / \mathrm{min}$ for $10 \mathrm{~min}$, and $2.5 \mathrm{~mL}$ of supernatant was absorbed. The residue was dried in a water bath at $37^{\circ} \mathrm{C}$ with nitrogen, dissolved in mobile phase (acetonitrile: water $=0.5: 99.5$ ) at $200 \mu \mathrm{L}$, mixed, and centrifuged at $10000 \mathrm{r} / \mathrm{min}$ for $10 \mathrm{~min}$. Finally, we took $10 \mu \mathrm{L}$ of the supernatant for HPLC analysis to calculate the concentration of catalpol in the plasma sample or brain tissue samples.

We used the following formula to evaluate the local bioavailability (F) and brain targeting index (DTI) of catalpol in plasma and brain tissue after intraintranasal of catalpol:

$\mathrm{F} \%=\mathrm{AUC}_{\text {i.n. } /} \mathrm{AUC}_{\text {i.v. }}{ }^{* 100 \%}$

$\mathrm{DTI}=\left(\mathrm{AUC}_{\text {brain }}\right.$ i.n. $/ \mathrm{AUC}_{\text {plasma }}$ i.n. $) /\left(\mathrm{AUC}_{\text {brain }}\right.$ i.v. $/$ $\left.\mathrm{AUC}_{\text {plasma i.v. }}\right)$

AUC refers to the area under curve; i.n. means intranasal administration; i.v. means intravenous administration. And DTI $>1$ indicates that the drug has brain targeting in brain tissue regions.

\section{The MCAO Model}

The MCAO model was performed according to a previously published protocol. ${ }^{19}$ The rats were anesthetized, the right common carotid (CCA), the right external carotid (ECA), and the right internal carotid (ICA) were carefully separated and exposed. The right external carotid and the right common carotid were ligated with a suture silk. Thereafter, a silicone-coated 4-0 nylon suture was gently advanced approximately $18-20 \mathrm{~mm}$ from the incision near the ECA-CCA branch. Nylon suture was advanced into the ICA lumen to block MCA blood flow until rats were sacrificed. During this procedure, the body temperature was maintained at $37^{\circ} \mathrm{C}$ using a warm pad. For the sham-operated group, only skin incisions were performed under anesthesia. After closure of the operative sites, the animals were allowed to awake from the anesthesia. After $72 \mathrm{~h}$ from the beginning of MCAO, rats were sacrificed. The SD rats were randomly divided into five groups: sham, model and catalpol groups $(2.5 \mathrm{mg} / \mathrm{kg}$, $5 \mathrm{mg} / \mathrm{kg}, 10 \mathrm{mg} / \mathrm{kg}$ ). Catalpol intranasal administration was described previously. Next, the animals were placed in cages and allowed free access to food and water.

\section{Measurement of Neurological Behavior}

The neurologic behavior was blindly evaluated using the mNSS. The neural behaviors of sham, model and catalpol groups were evaluated at $24 \mathrm{~h}, 48 \mathrm{~h}$ and $72 \mathrm{~h}$. mNSS is a composite of the motor (muscle status, abnormal movement), sensory (visual, tactile, and proprioceptive), and systematically evaluate the severity of nerve injury. Neurological function was graded on a scale of 0 to 18 (normal score 0; maximal deficit score 18). A score of 1318 indicates severe injury, a score of 7-12 indicates moderate injury, and a score of 1-6 indicates mild injury. One point is awarded for each abnormal behavior or for lack of a tested reflex; thus, the higher the score, the more severe the injury. ${ }^{20}$

\section{Infarct Volume Evaluation}

After $72 \mathrm{~h}$ from the beginning of MCAO, rats were euthanized by decapitation and brains were cut into $2 \mathrm{~mm}$ thick of 5 coronal slices. The slices were stained with $0.5 \%$ 2,3,5-triphenyltetrazolium chloride (TTC) for $0.5 \mathrm{~h}$ at $37^{\circ} \mathrm{C}$. Unstained areas were defined as infarcts and measured using microscope image-analysis software (Image-Pro Plus, U.S.A.). The percentage of the infarct volume was calculated by the following formula: Infarct volume $(\%)=$ (normal hemisphere volume - noninfarct volume of the infarct side)/normal hemisphere volume*100\%.

\section{Brain Water Content Analysis}

The rats were decapitated $72 \mathrm{~h}$ after MCAO and sacrificed for brain collection. After the wet weight is weighed, the dry weight is weighed after drying in the oven at $105^{\circ} \mathrm{C}$ to a constant weight. $\% \mathrm{H}_{2} \mathrm{O}$ was obtained as (Brain wet weight - Brain dry weight)/Brain wet weight*100\%.

\section{Determination of Oxidative Stress}

The rats were decapitated $72 \mathrm{~h}$ after MCAO. Blood samples were collected and serum samples were collected after centrifugation at $1800 \mathrm{rpm} 10 \mathrm{~min}$ for testing. The SOD and MDA contents were assessed by assay kits from Nanjing Jiancheng Bioengineering Institute following the manufacturer's instruction. The absorbance was determined with a microplate reader.

\section{Hematoxylin-Eosin (HE) and TUNEL Staining}

Rats were deeply anesthetized after $72 \mathrm{~h}$ after MCAO, perfused with physiological saline and $4 \%$ paraformaldehyde and then decapitated. Brains were dehydrated and embedded in paraffin and then cut into $4 \mu \mathrm{m}$ coronal 
sections; subsequently, brain sections were deparaffinized and hydrated for HE and TUNEL staining. Neuronal apoptosis was detected with the terminal deoxynucleotidyl transferase (TdT)-mediated dUTP nick end labeling (TUNEL) using a cell death detection kit following the manufacturer's instructions.

\section{Western Blotting}

Western blotting and cellular fractionation were performed as previously described. ${ }^{21}$ Briefly, the periinfarction brain cortex was lysed on ice in lysis buffer [50 mm Tris- $\mathrm{HCl}(\mathrm{pH} 8.2), 0.5 \mathrm{M}$ saccharose, $10 \mathrm{mM}$ HEPES (pH7.9), $1.5 \mathrm{mM} \mathrm{MgCl} 2,10 \mathrm{mM} \mathrm{KCl}, 1 \mathrm{mM}$ EDTA, 10\% (v/v) glycerin, 1 mM DTT, 1 mM PMSF, 10 $\mu \mathrm{g} / \mathrm{mL}$ Aprotinin, and $5 \mu \mathrm{g} / \mathrm{mL}$ Leu-peptin]. After centrifugation at $16,000 \mathrm{r} / \mathrm{min}$ for $10 \mathrm{~min}$, the protein content in cleared lysate was determined by the Bradford assay. Lysate samples containing $40 \mu \mathrm{g}$ of protein were fractio-nated by $10 \%$ SDS-polyacrylamide gel electrophoresis and then electroblotted onto PVDF membranes. The following antibodies were used at the indicated concentrations: rabbit anti-Nrf2 (1:1000; Proteintech; 16396-1-AP), rabbit anti-HO-1 (1:1000; Proteintech; 10701-1-AP), rabbit anti-Bcl-2 (1:1000; Proteintech; 12789-1-AP), rabbit anti-Bax (1:2000; Proteintech; $50599-2-1-\lg )$, rabbit anti- $\beta$-actin (1:2000; Proteintech; 20536-1-AP). The immunoreactive bands were digitally scanned using the ScanMaker E6 system and quantified using a UVP gel imaging system and the Labworks 4.6 software (Amersham, American). The $\beta$-actin was used as an internal control for all Western blots.

\section{Molecular Docking Analysis}

Molecular docking analysis between catalpol and were performed using Discovery Studio 3.5. The structures of Nrf2, HO-1, Bcl-2, BAX were obtained from the Protein Data Bank (PDB) archives and used as target for molecular docking. The structure of catalpol was drawn by ChemDraw.

\section{Statistical Analysis}

All data analyses were performed using GraphPad Prism 8 (GraphPad software, San Diego, CA). The experimental results were presented as means $\pm \mathrm{SD}$. Data from different groups were compared using one-way ANOVA. In all statistical analysis, $P<0.05$ was considered to be statistically significant.

\section{Results}

\section{Hemolysis Test and Ciliotoxicity Test Shown Catalpol is Safety via Nasal} Administration

No hemolysis was observed in any concentration of catalpol. The PVD of cilia is shown in Figure 1A, and the $\mathrm{PPV}$ is shown in Figure 1B. Usually, the percentage of continuous movement time of cilia is more than $50 \%$, which means that the drug does not affect the ability of cilia to move. The results showed that the PVD of cilia in the deoxysodium cholate group was only 6 minutes, and the PPV was only $1.2 \%$. It showed that deoxysodium cholate was severely toxic to ciliary movement. The PPV of the catalpol group was $83 \%$. The PVD of the catalpol group was 404 minutes; the results showed that catalpol had little effect on nasal ciliary movement.

The results of HE staining of the nasal mucosa of rats are shown in Figure 1C. In normal saline group, the structure of the nasal mucosa of rats was completed, the cells were arranged in an orderly way, and the nuclei were of the same regular size. No abnormal structures were observed. In the deoxysodium cholate group, the obtained photomicrographs demonstrated that the structure of the nasal mucosa was obviously incomplete, epithelial cells in most areas necrotic and sparse, and the olfactory epithelium exhibited seriously degenerative variations. It was confirmed that sodium deoxycholate has serious toxicity to nasal mucosa. In catalpol group, the olfactory epithelium exhibited slight degenerative variations and the lamina propria had reasonable infiltrations of mononuclear cells without any severe degenerative changes. Consequently, the safety of the catalpol intranasal administration was assured.

\section{Method Validation Shown That Specificity, Linearity, Accuracy, Precision, Recovery and Stability Specificity}

As shown in Figure 2, the retention time of catalpol and internal standard were $6.7 \mathrm{~min}$ and $14.3 \mathrm{~min}$ respectively, and the analysis time of each sample was $20 \mathrm{~min}$. The endogenous substances in plasma and brain tissue samples did not interfere with catalpol and internal standard and had strong specificity.

\section{Linearity}

The standard curves of plasma and brain tissue are shown in Table 1. The plasma catalpol showed a good linear 
A

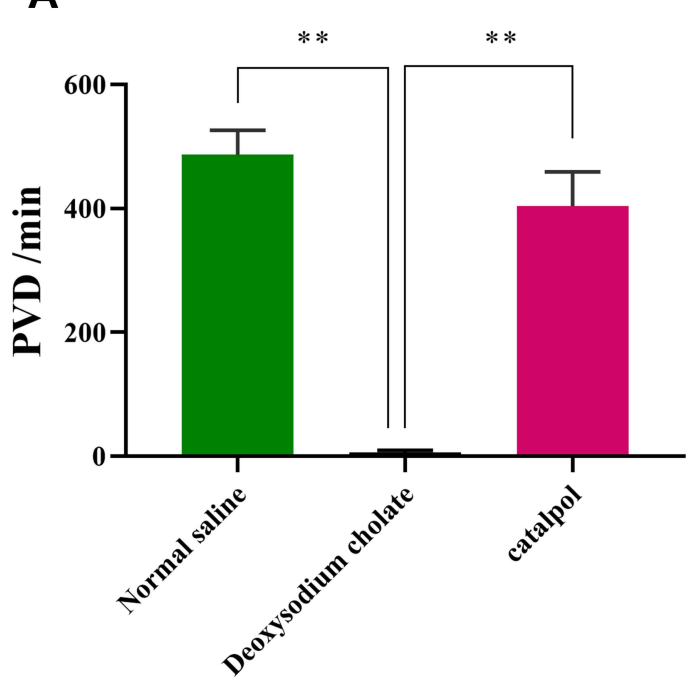

B

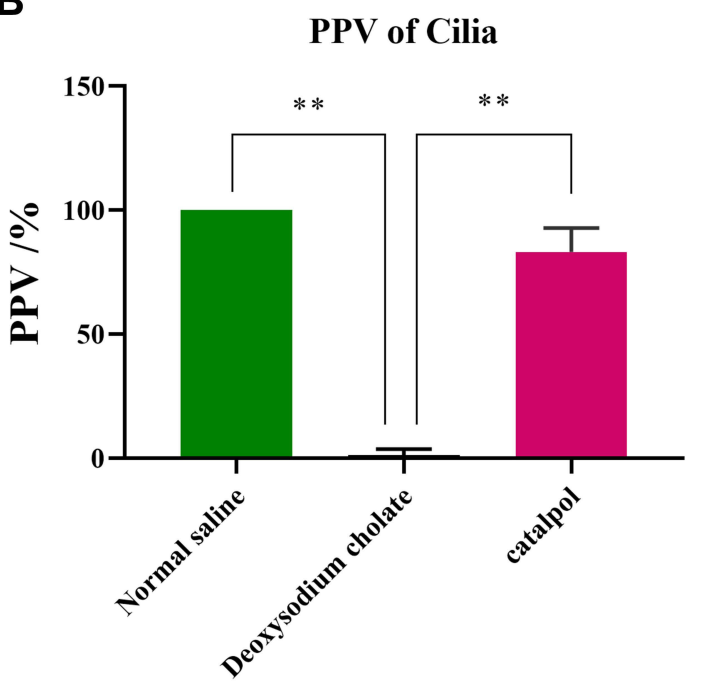

C

1 day
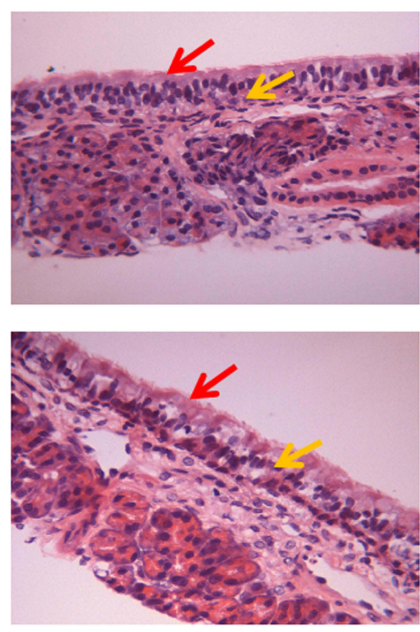

4 day

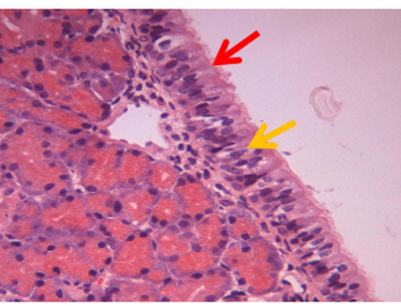

Stop 7 day

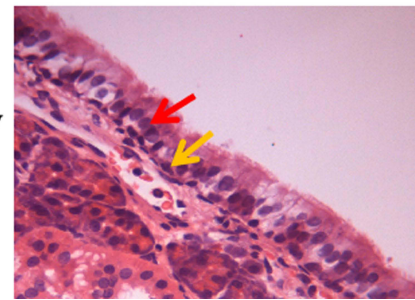

Deoxysodium cholate
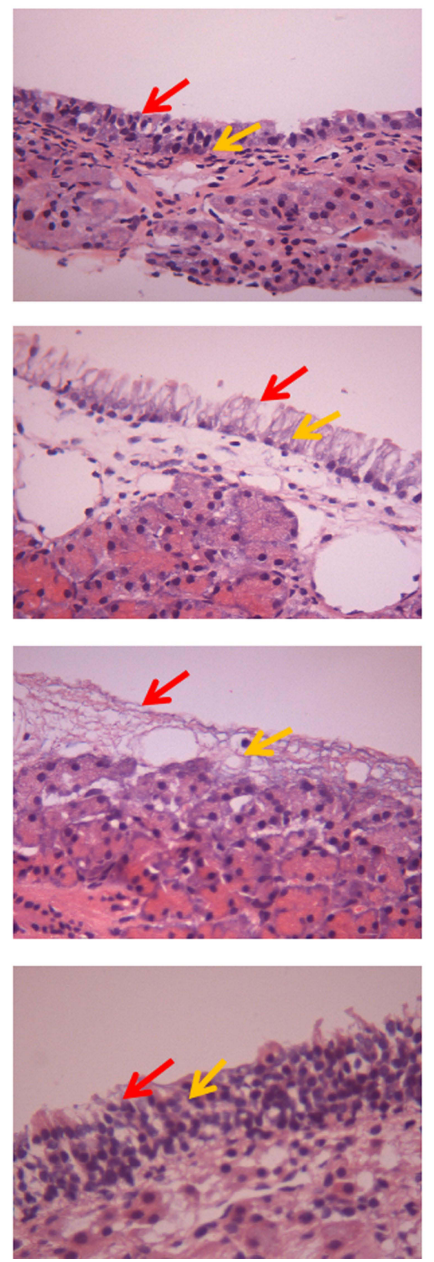

Catalpol
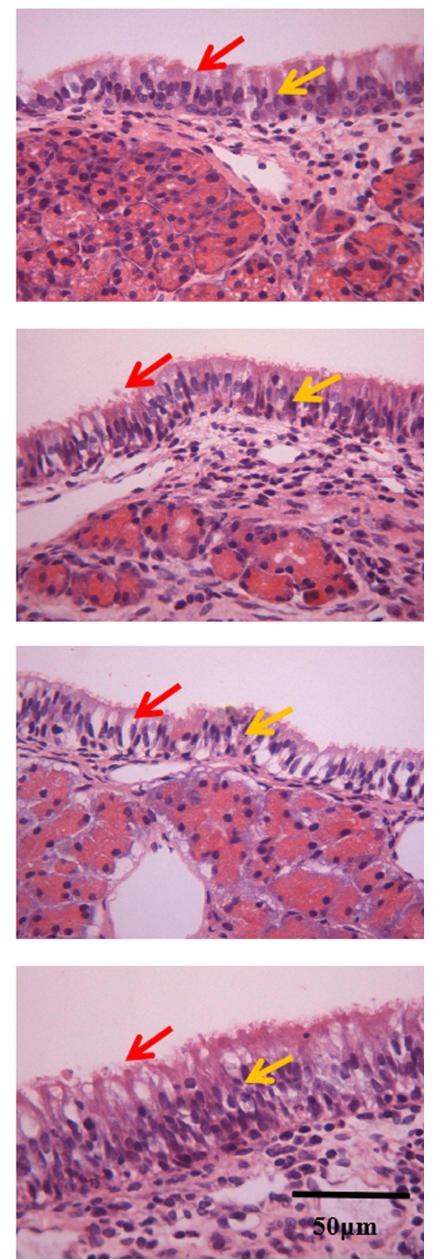

Figure I Hemolysis test and ciliotoxicity test shown catalpol is safety via nasal administration (A) the PVD of cilia; (B) the PPV of cilia; (C) damage of catalpol nasal drops on nasal mucosa of rats $(400 \times, n=3, " \longrightarrow$ "indicates nasal cilia," $\longrightarrow$ ” indicates epithelial cell). you may use the arrow to show the effects (the data are presented as the mean \pm S.D., $\mathrm{n}=3, * * p<0.01)$. 


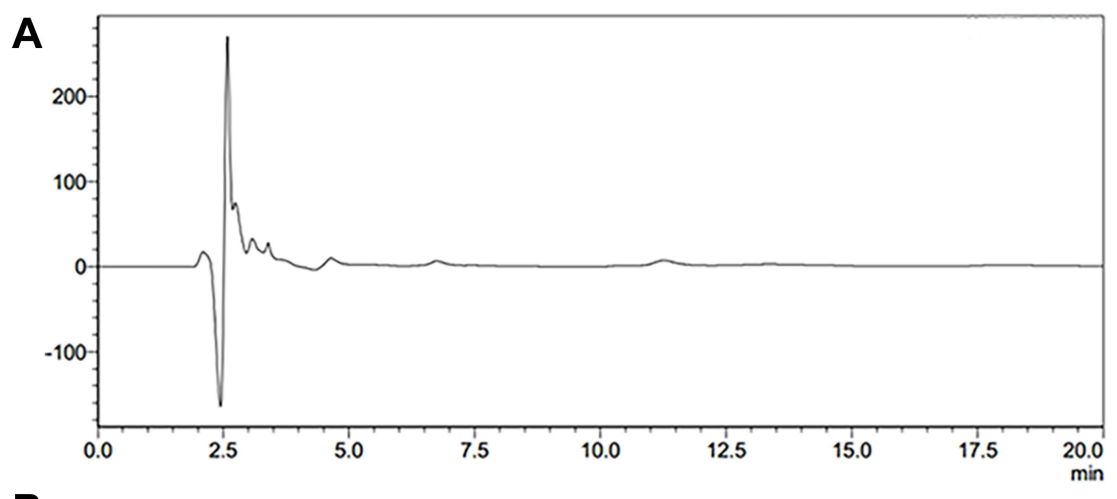

B

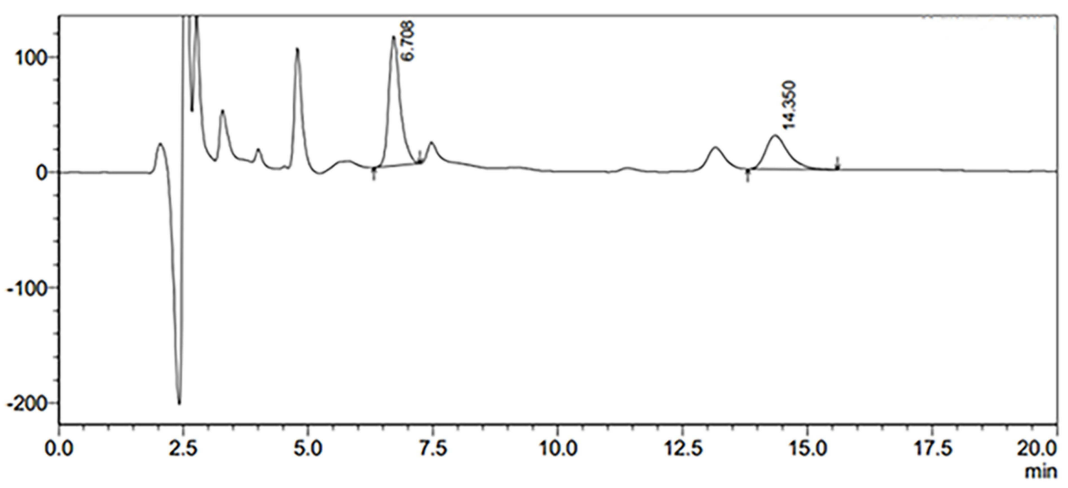

C

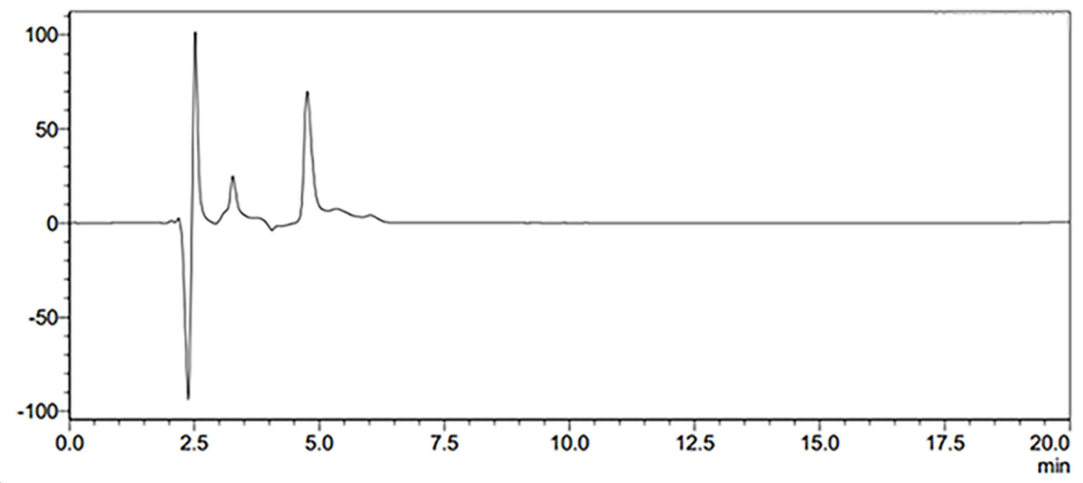

D

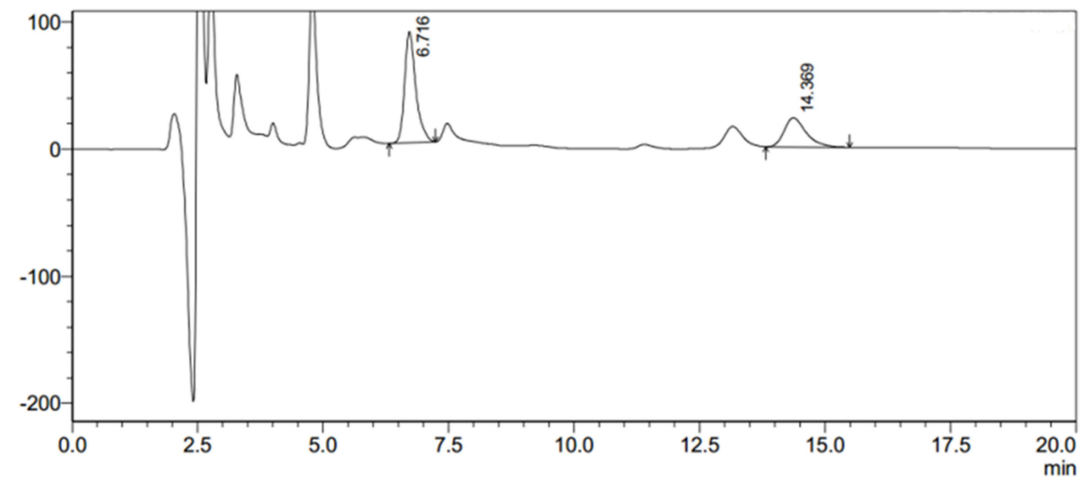

Figure 2 (A) Blank brain samples; (B) catalpol and the internal standard in brain samples; (C) blank plasma; (D) catalpol and the internal standard in blank plasma. 
Table I Standard Curve Derived from Catalpol Samples of Plasma and Brain $(n=3)$

\begin{tabular}{|c|c|c|c|}
\hline Sample & Standard Curve & $\mathbf{r}$ & $\begin{array}{l}\text { Quantitative } \\
\text { Range }\end{array}$ \\
\hline Plasma $(\mu \mathrm{g} / \mathrm{mL})$ & $y=1.5878 x-2.0624$ & 0.9951 & $1 \sim 1000$ \\
\hline Brain $(\mu g / g)$ & $y=0.6959 x-0.8163$ & 0.9913 & $0.5 \sim 50$ \\
\hline
\end{tabular}

relationship in the quantitative range of $1-1000 \mu \mathrm{g} / \mathrm{mL}$, and the limit of quantification was $1 \mu \mathrm{g} / \mathrm{mL}$. The quantitative of catalpol in brain tissue was linear in the range of $0.5-50 \mu \mathrm{g} / \mathrm{g}$, and the limit of quantification was 0.5 $\mu \mathrm{g} / \mathrm{mL}$.

\section{Precision Stability and Recovery}

As shown in Table 2, this method has good precision and reliability. The stability test results of catalpol in rat plasma and brain homogenate tissue are shown in Table 3. The results showed that catalpol was stored at $-80^{\circ} \mathrm{C}$ for 20 days (RSD $<8.64 \%$ ) and three freeze-thaw cycles (RSD $<6.99 \%$ ), and the method had good stability.

The recovery results (Table 4 ) showed that the recovery rate of catalpol in plasma samples and brain samples with low, medium and high concentration was more than

Table 2 Precision of Catalpol in Rat Plasma and Brain $(n=5$, Mean \pm SD)

\begin{tabular}{|l|l|l|l|}
\hline Sample & $\begin{array}{l}\text { Added } \\
\text { Concentration }(\mu \mathrm{g} / \mathrm{mL})\end{array}$ & $\begin{array}{l}\text { Measured } \\
(\mu \mathrm{g} / \mathrm{mL})\end{array}$ & $\begin{array}{l}\text { RSD } \\
(\%)\end{array}$ \\
\hline Plasma $(\mu \mathrm{g} / \mathrm{mL})$ & 1 & $0.97 \pm 0.12$ & 12.09 \\
& 10 & $9.06 \pm 0.82$ & 9.09 \\
& 100 & $97.10 \pm 1.92$ & 1.97 \\
\hline Brain $(\mu \mathrm{g} / \mathrm{g})$ & 1 & $0.96 \pm 0.14$ & 14.28 \\
& 10 & $9.20 \pm 1.23$ & 13.41 \\
& 100 & $92.16 \pm 1.84$ & 1.99 \\
\hline
\end{tabular}

Table 3 Stability of Catalpol in Rat Plasma and Brain $(n=5)$

\begin{tabular}{|l|l|l|l|}
\hline \multirow{2}{*}{ Sample } & \multirow{2}{*}{$\begin{array}{l}\text { Added } \\
\text { Concentration } \\
\end{array}$} & $(\mu \mathrm{g} / \mathrm{mL})$ & \multicolumn{2}{|l|}{ RSD (\%) } \\
\cline { 3 - 4 } & & $\begin{array}{l}\text { Long Term } \\
\text { Stability }\end{array}$ & $\begin{array}{l}\text { Freeze-Thaw } \\
\text { Cycle Stability }\end{array}$ \\
\hline Plasma & $\mathrm{I}$ & 8.64 & 6.43 \\
$(\mu \mathrm{g} / \mathrm{mL})$ & 10 & 5.83 & 5.19 \\
& 100 & 5.12 & 6.99 \\
\hline Brain & 1 & 7.89 & 6.74 \\
$(\mu \mathrm{g} / \mathrm{g})$ & 10 & 2.67 & 5.73 \\
& 100 & 4.96 & 3.82 \\
\hline
\end{tabular}

Table 4 Recovery of Catalpol in Rat Plasma and Brain $(n=3$, Mean \pm SD)

\begin{tabular}{|l|l|l|l|}
\hline Sample & $\begin{array}{l}\text { Added Concentration } \\
(\mu \mathrm{g} / \mathrm{mL})\end{array}$ & Recovery (\%) & RSD (\%) \\
\hline $\begin{array}{l}\text { Plasma } \\
(\mu \mathrm{g} / \mathrm{mL})\end{array}$ & 5 & $89.15 \pm 9.7$ & 10.88 \\
& 1000 & $96.17 \pm 10.68$ & 11.10 \\
& $91.22 \pm 15.66$ & 17.16 \\
\hline $\begin{array}{l}\text { Brain } \\
(\mu \mathrm{g} / \mathrm{g})\end{array}$ & 1 & $87.67 \pm 12.73$ & 14.52 \\
& 50 & $90.93 \pm 8.42$ & 9.25 \\
& $85.98 \pm 7.06$ & 8.21 \\
\hline
\end{tabular}

$85.98 \%$, which met the requirements of biological sample analysis and indicated that the determination method had a good recovery rate.

\section{Catalpol Intranasal Has Well Brain Targeting and Pharmacokinetics}

The distribution results of catalpol concentration in plasma after intranasal (i.n.) and intravenous (i.v.) are shown in Figure 3A. The concentration of catalpol in plasma after intranasal was much lower than intravenous. However, the absorption was faster after i.n., and could be detected in plasma at $15 \mathrm{~min}$, and maintained at a certain concentration for a certain period. Catalpol was detected in all regions of the brain tissue 15 minutes after intranasal and intravenous. The results of its distribution in the olfactorius bulbus, hippocampus, medulla oblongata, the cerebellum and cortexol are shown in Figure 3B-F.

The concentration of catalpol during intranasal was higher in all areas of brain tissue than intravenous. DAS software was further used to calculate the pharmacokinetic parameters of brain tissue regions under different administration routes. After intranasal, the AUC of catalpol in the olfactorius bulbus, hippocampus, medulla oblongata, the cerebellum and cortexol was significantly higher than intravenous (Table 5). These results showed that catalpol was absorbed rapidly through intranasal, and its concentration in brain tissue is significantly higher than intravenous.

We calculated the brain targeting index of catalpol in rat brain tissue after intranasal by formula (Table 6). The DTI of catalpol in the olfactorius bulbus, hippocampus, medulla oblongata, the cerebellum and cortexol were 7.99, $9.10,28.65,13.15$, and 14.50 , respectively, which were all higher than 1, suggesting that the catalpol intranasal administration has good brain targeting. 


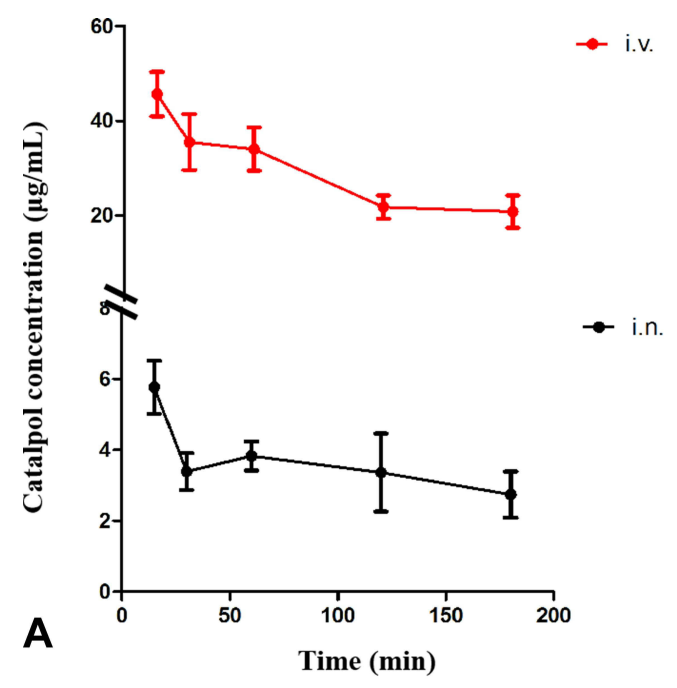

Hippocampus
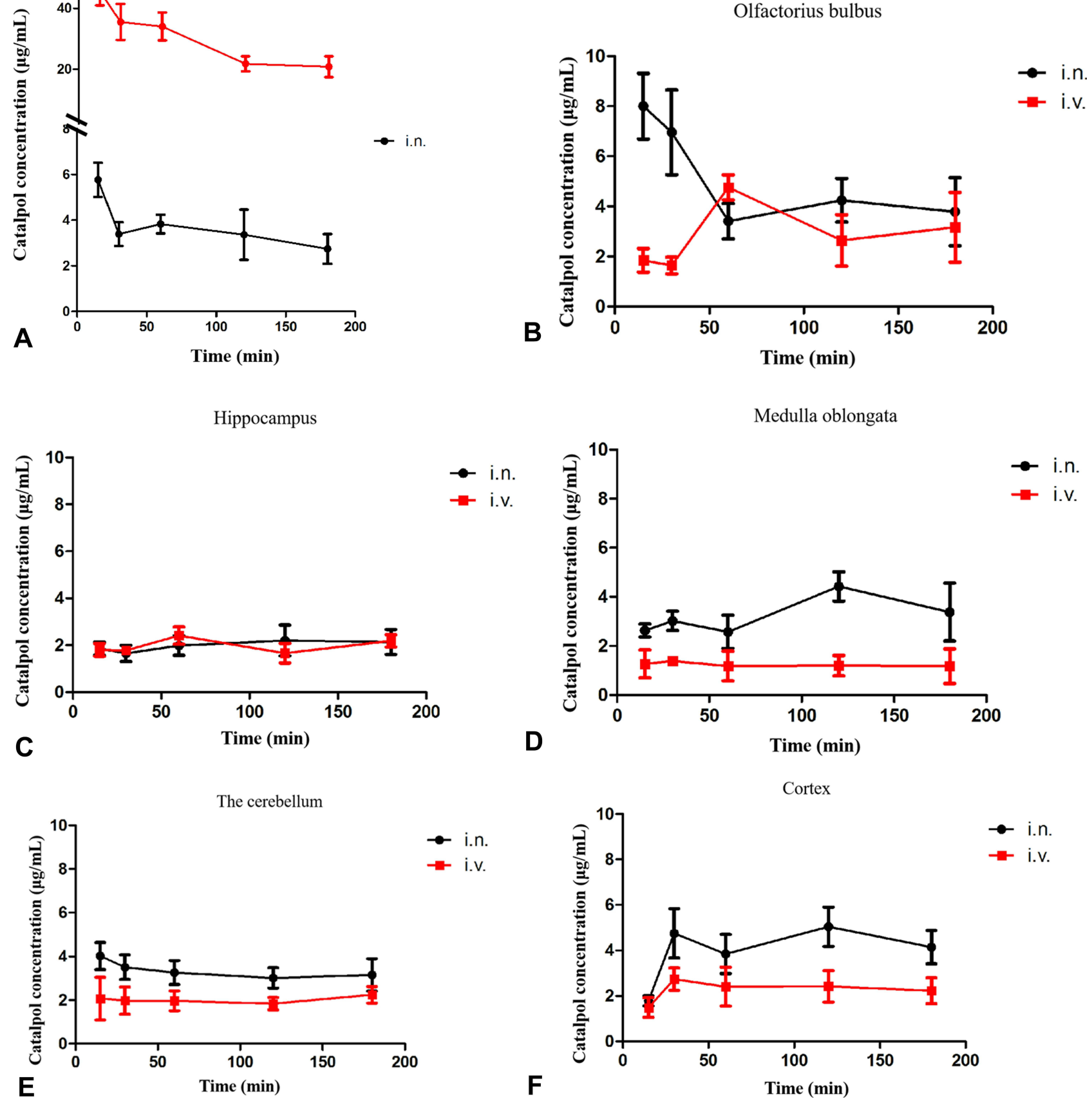

Figure 3 (A) Catalpol concentration in plasma after nasal and intravenous; (B-F) catalpol concentration in each tissue region after intranasal and intravenous (i.n. intranasal, i.v. intravenous).

\section{Catalpol Intranasal Administration Reduced Neurological Dysfunction and Infarct Volume}

To examine the efficacy of catalpol intranasal administration on cerebral ischemic injury. Rats were given intranasal administration for 3 days after MCAO. To observe the protective effect of catalpol intranasal administration on cerebral ischemia injury. Compared with the sham group, the mNSS score in the model group caused was obviously increased $(P<0.01)$. The score of mNSS in $24 \mathrm{~h}, 48 \mathrm{~h}$, and $72 \mathrm{~h}$ was increased in the catalpol groups (Figure $4 \mathrm{~A}-\mathrm{C}$ ). TTC staining was used to measure the infarct volume in the ischemic area. Compared with sham group, model group injury increased by $26.69 \%$, whereas intranasal of 
Table 5 Pharmacokinetic Parameters of Catalpol After Intranasal and Intravenous $(n=3)$

\begin{tabular}{|l|l|l|l|l|l|l|}
\hline \multirow{2}{*}{ Tissue } & \multicolumn{2}{l}{ in } & \multicolumn{2}{l}{ iv } \\
\cline { 2 - 7 } & Cmax (ug/mL) & Tmax (min) & AUC & Cmax (ug/mL) & Tmax (min) & AUC \\
\hline Plasma & 5.8 & 15 & 618.975 & 45.7 & 15 & 4947.356 \\
Olfactorius bulbus & 4.8 & 60 & 633.031 & 4.8 & 60 & 60 \\
Hippocampus & 2.9 & 15 & 388.475 & 2.4 & 30 & 33.048 \\
Medulla oblongata & 4.4 & 180 & 499.068 & 1.4 & 180 & 139.22 \\
The cerebellum & 4.0 & 15 & 561.265 & 2.2 & 327 \\
Cortex & 5.0 & 120 & 733.582 & 2.7 & 341.117 \\
\hline
\end{tabular}

Table 6 Intranasal Catalpol Brain Targeting Index and Bioavailability Histopathological Study $(n=3)$

\begin{tabular}{|l|l|l|}
\hline Tissue & F (\%) & DTI \\
\hline Plasma & 0.12 & - \\
Olfactorius bulbus & 1.00 & 7.99 \\
Hippocampus & 1.14 & 9.10 \\
Medulla oblongata & 3.58 & 28.65 \\
The cerebellum & 1.64 & 13.15 \\
Cortex & 1.81 & 14.50 \\
\hline
\end{tabular}

catalpol obviously reduced the infarct volume by MCAO (Figure 4D and E). HE staining results showed that compared with the sham group, in the model group the number of cells was reduced, the arrangement was irregular, and the karyotype changed significantly. To a certain extent, intranasal administration of catalpol reversed the pathological changes, showing that intranasal catalpol had a protective effect on cerebral ischemia injury (Figure 5A).

\section{Catalpol Intranasal Administration Mitigated Brain Edema}

As shown in Figure 5C, the water level in the brains of the model group was remarkably greater than that of the sham group $(p<0.01)$, indicating that brain edema was evident after MCAO. However, the water content in the brains of catalpol was remarkably less than that of the model group $(p<0.01)$, suggesting that catalpol via intranasal administration reduced the brain edema caused by ischemic injury.

\section{Catalpol Intranasal Administration Reduces Apoptosis}

TUNEL staining was used to determine cell death (Figure 6A). The number of dying cells was notably augmented in the cortex of the model group compared with the sham group. Intranasal catalpol treatment markedly decreased the cell apoptosis in the infarct region $(p<0.01)$. In addition, we detected the expression of apoptotic-related proteins including Bcl-2 and Bax (Figure 6C). Consistent with TUNEL staining, catalpol via intranasal administration reduced the decrease of Bcl-2, the increase of Bax. These results indicated that catalpol via intranasal administration significantly inhibit apoptosis induced by cerebral ischemia.

\section{Catalpol Intranasal Administration Reduces Oxidative Damage and Induces the Expression of Nrf2, HO-I After MCAO}

Oxidative damage was assessed by measuring SOD and MDA content. The level of SOD in model group was significantly reduced and MDA content was significantly higher than sham group (Figure 7A and B). Intranasal administration of catalpol could significantly increase SOD content and reduced MDA content. We also detected the expression of oxidative-stress-related proteins $\mathrm{Nrf2}$ and HO-1 (Figure 7C-E). The results showed that intranasal administration of catalpol could significantly induces the expression of Nrf2, HO-1 in ischemic brain region. These results indicated that catalpol via intranasal administration significantly reduce oxidative damage.

\section{Molecular Docking Analysis Shown that Catalpol Has Certain Binding Effect with Nrf2, HO-I, Bcl-2, BAX}

The $3 \mathrm{D}$ and $2 \mathrm{D}$ binding graphs of the small molecule and the target protein after docking are shown in Figure 8. Results show that Nrf2 (lowest binding energy: -6.96 $\mathrm{kcal} / \mathrm{mol}$ ), HO-1 (lowest binding energy: $-4.64 \mathrm{kcal} /$ $\mathrm{mol}$ ), BAX (lowest binding energy: $-4.4 \mathrm{kcal} / \mathrm{mol}$ ) and Bcl-2 (lowest binding energy: $-3.87 \mathrm{kcal} / \mathrm{mol}$ ). The results showed that catalpol has certain binding effect with Nrf2, HO-1, Bcl-2, BAX, mainly through hydrogen bonding. 
A

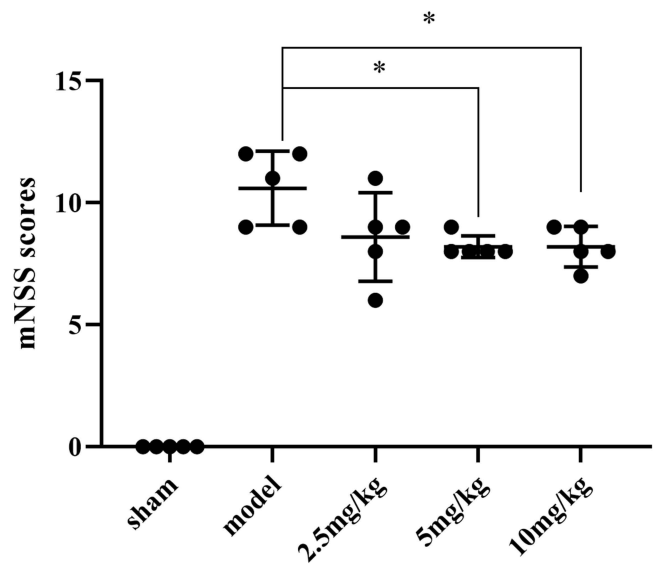

C

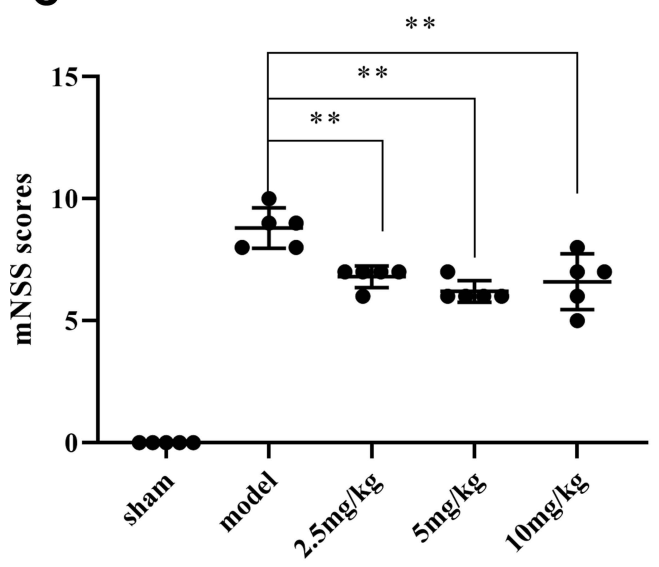

B

$48 h$

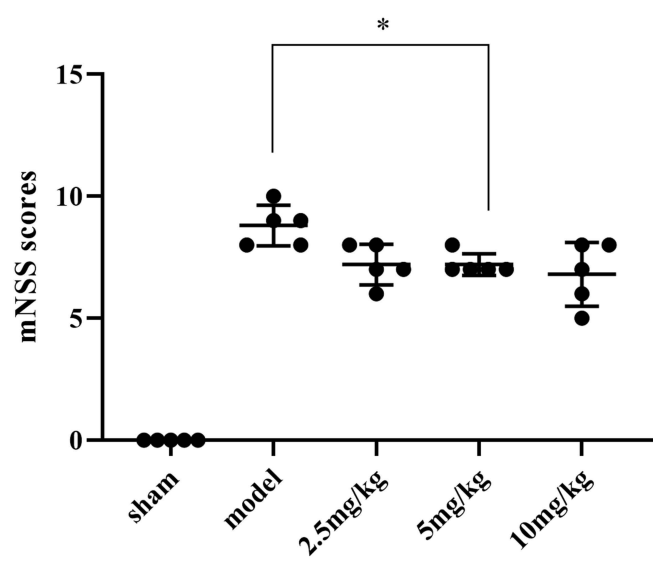

D

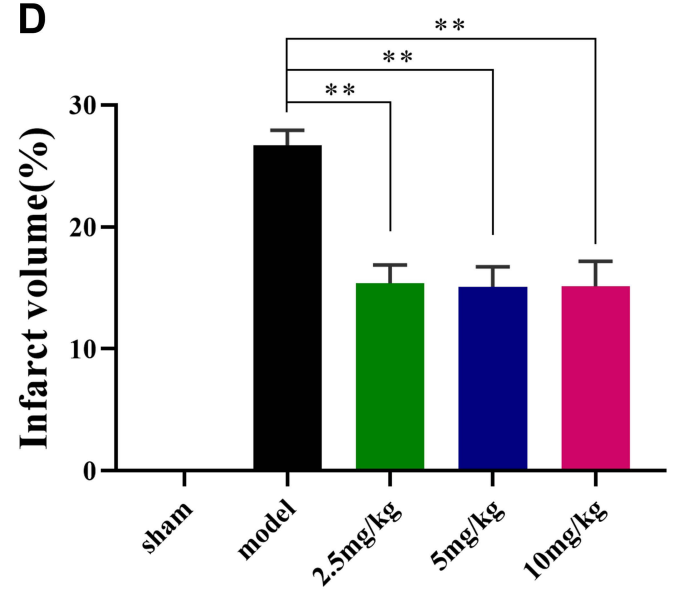

E

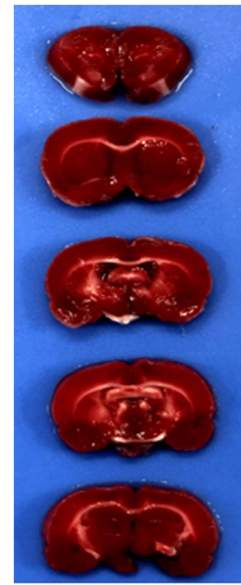

Sham

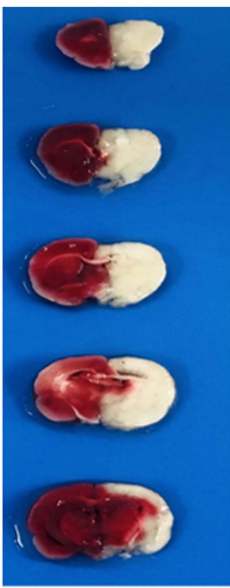

Model

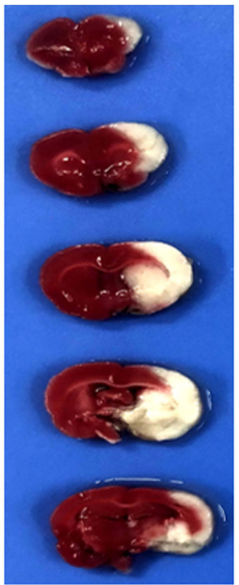

2.5

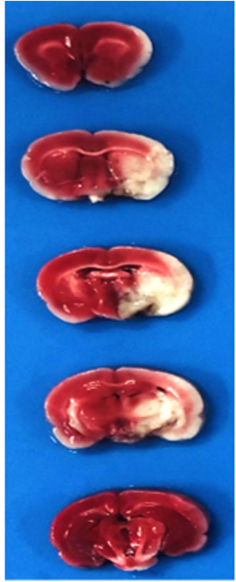

5

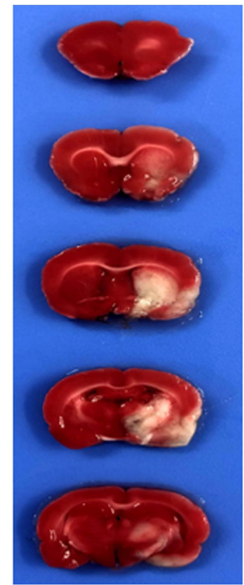

10

Catalpol(mg/kg)

Figure 4 Catalpol protected in MCAO mice. (A) Modified neurological severity scores in $24 \mathrm{~h}$ after ischemia; (B) modified neurological severity scores in 48 after ischemia; (C) modified neurological severity scores in 48 after ischemia (the data are presented as the mean \pm S.D., $n=5$, $* p<0.05$, **p $<0.01$ ); (D) the percentage of infarct volume was detected for each group; (E) representative TTC staining of the cerebral infarct in brain (the data are presented as the mean \pm S.D., $n=3$, $* * p<0.0 \mathrm{I}$ ). 
A
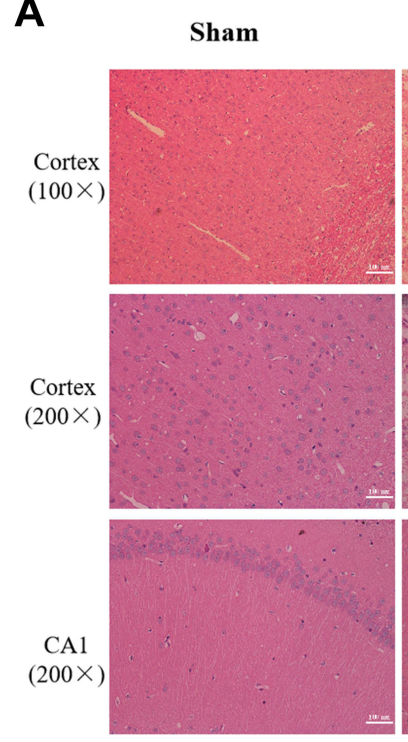

\section{B}

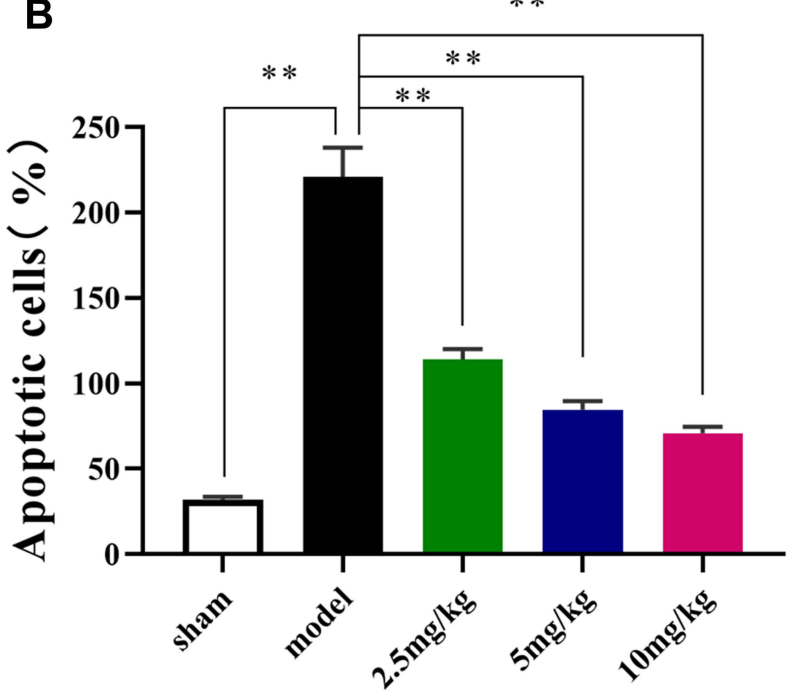

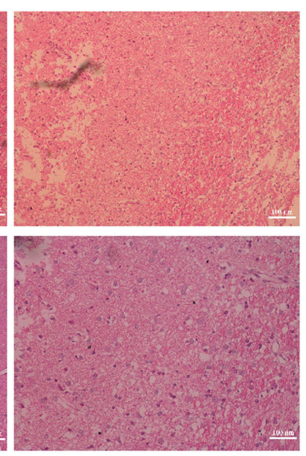

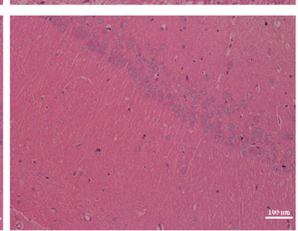

Model
Catalpol(mg/kg)

2.5
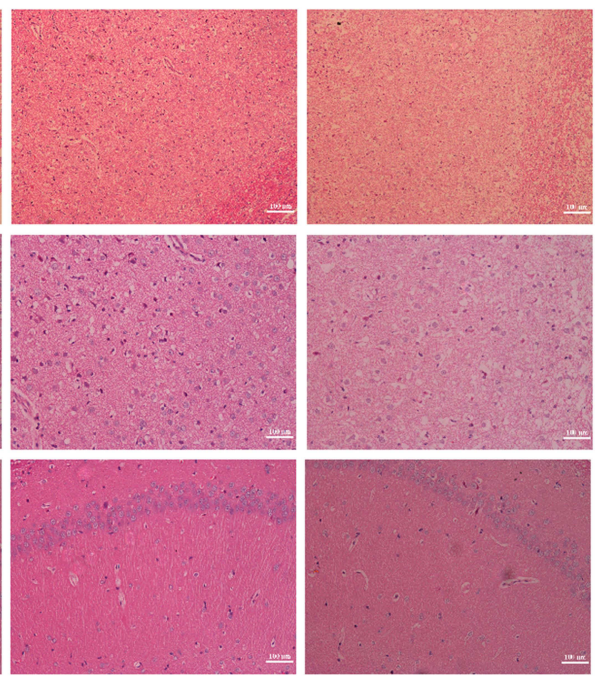

10
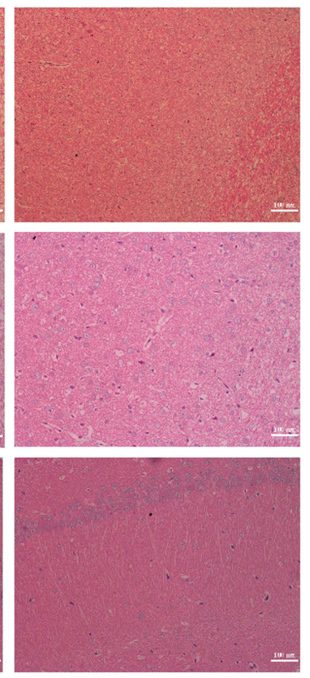

C

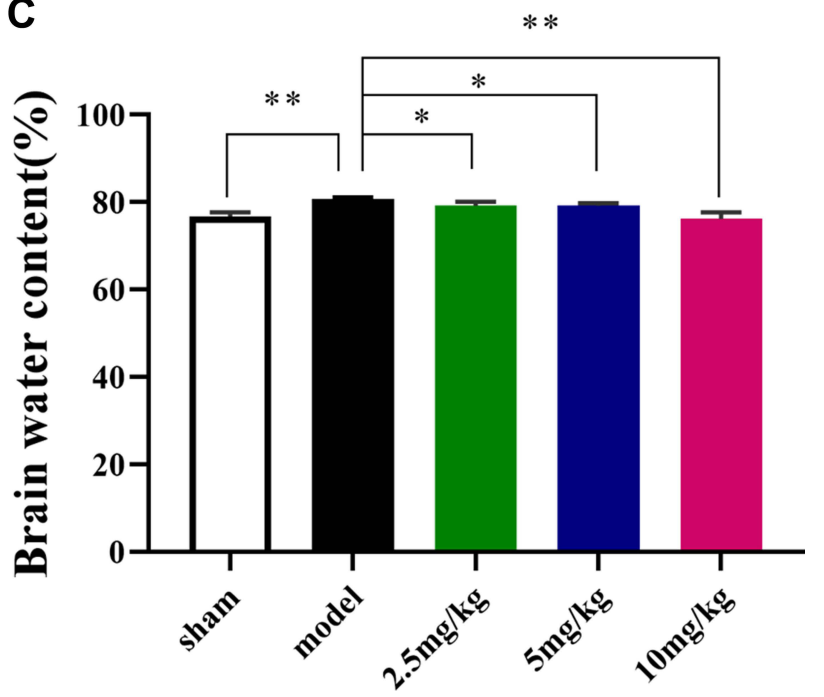

Figure $\mathbf{5}$ (A and B) HE staining performed on sections from ischemic cortex and CAI, $\mathrm{n}=3$ per group; (C) brain water content (the data are presented as the mean \pm S.D., $\left.\mathrm{n}=5, * p<0.05, * *_{p}<0.01\right)$.

\section{Discussion}

Rehmannia Decoction is a representative prescription, which is an effective prescription for the treatment of ischemic stroke. ${ }^{22}$ Its efficacy in the treatment of ischemic stroke has been confirmed by clinical and experimental studies, and it is still used in the clinical treatment of cerebral apoplexy. ${ }^{23}$ Catalpol is not only the main active component of Rehmannia glutinosa, but also the main active component. ${ }^{24}$ Our previous studies have shown that catalpol has an obvious neuroprotective effect on brain injury caused by cerebral ischemia. Prophylactic administration, 6h, 24h therapeutic administration has a therapeutic effect, and there is a longer treatment time window. Catalpol can promote angiogenesis in the ischemic brain area, protect cerebral neurovascular units, and promote the recovery of neurological function in stroke rats. $^{22,25}$

Central Nervous System (CNS) diseases include stroke, brain tumor, CNS infection, migraine, etc. The lesion is located in the brain, but due to the existence of blood-brain barrier (BBB), many drugs cannot be administered in sufficient effective amount to the brain. As a result, the drug concentration is low, which affects the treatment effect of brain disease. ${ }^{26}$ The nasal cavity is the only part of the brain that can communicate with the outside world, and its unique anatomical structure makes it 

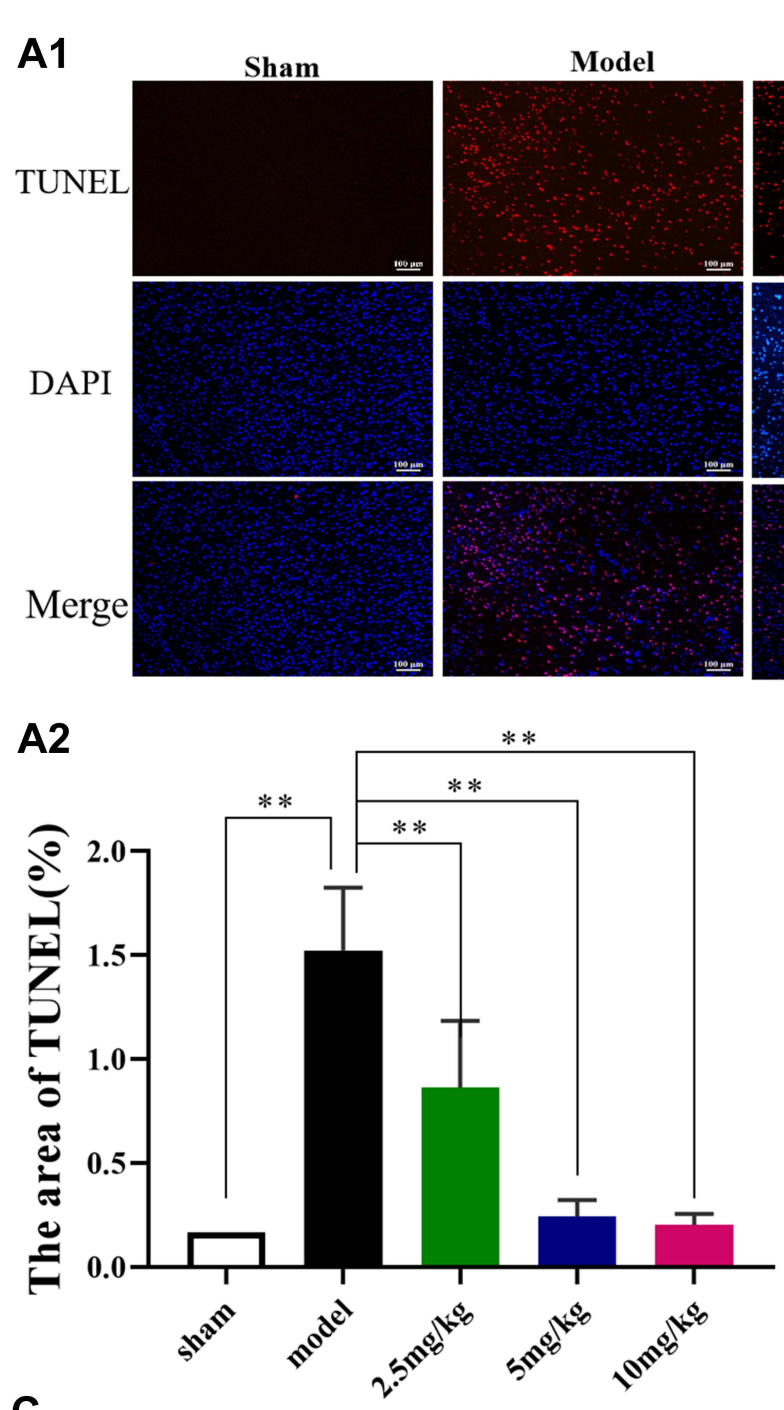

C

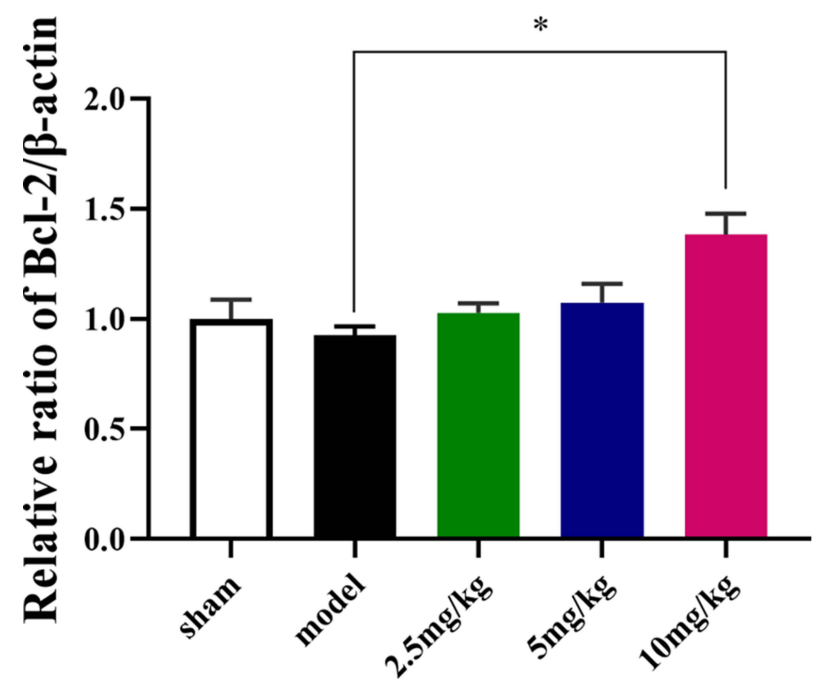

Catalpol(mg/kg)

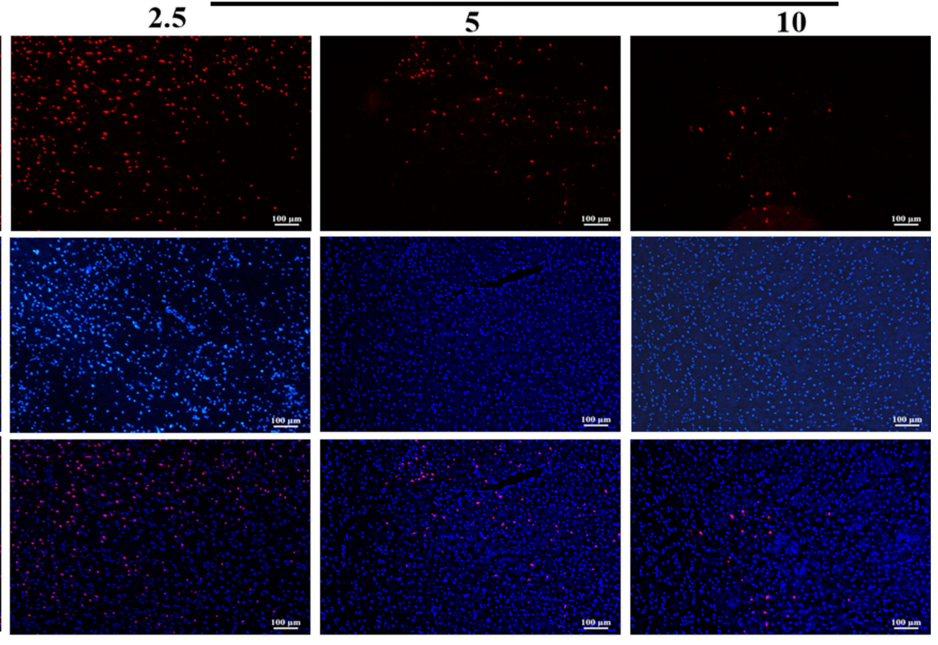

B
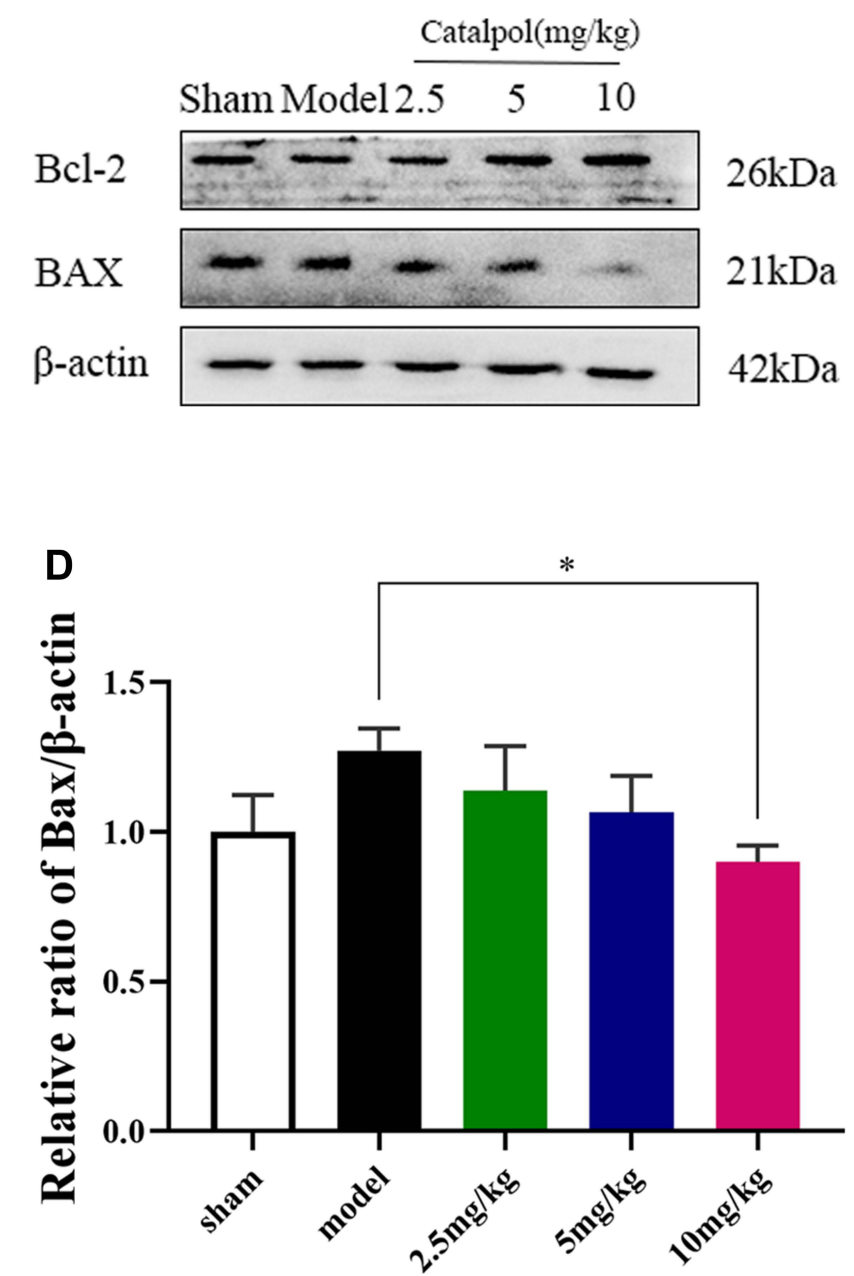

Figure 6 Catalpol intranasal administration reduces apoptosis. (A) TUNEL staining performed on sections from ischemic cortex; (B) the activating effect of catalpol on the expression of $\mathrm{Bcl}-2$ pathway-related proteins; (C) the protein expression levels of $\mathrm{Bcl}-2$; (D) the protein expression levels of Bax (the data are presented as the mean \pm S.D., $\left.\mathrm{n}=3,{ }^{*} p<0.05, * *_{p}<0.01\right)$ 

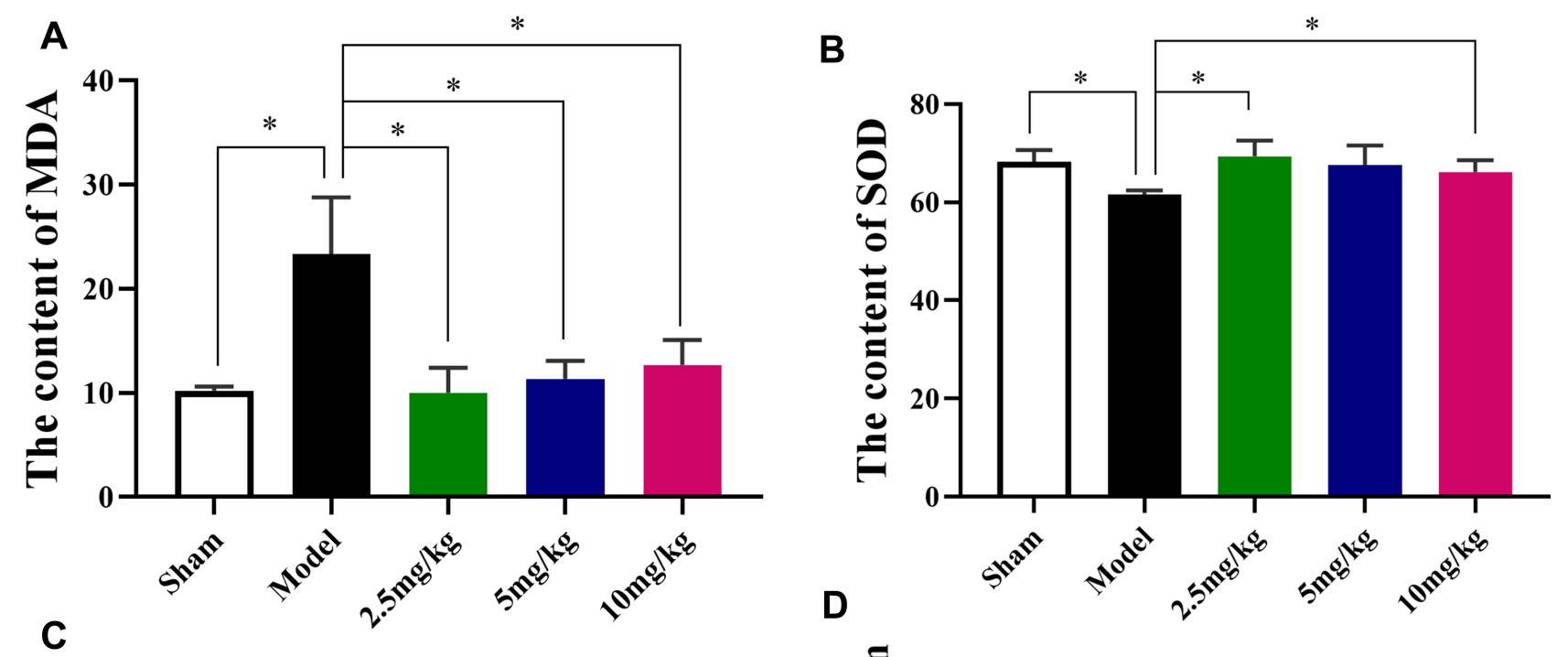

Catalpol(mg/kg)

Sham Model $2.5 \quad 5 \quad 10$
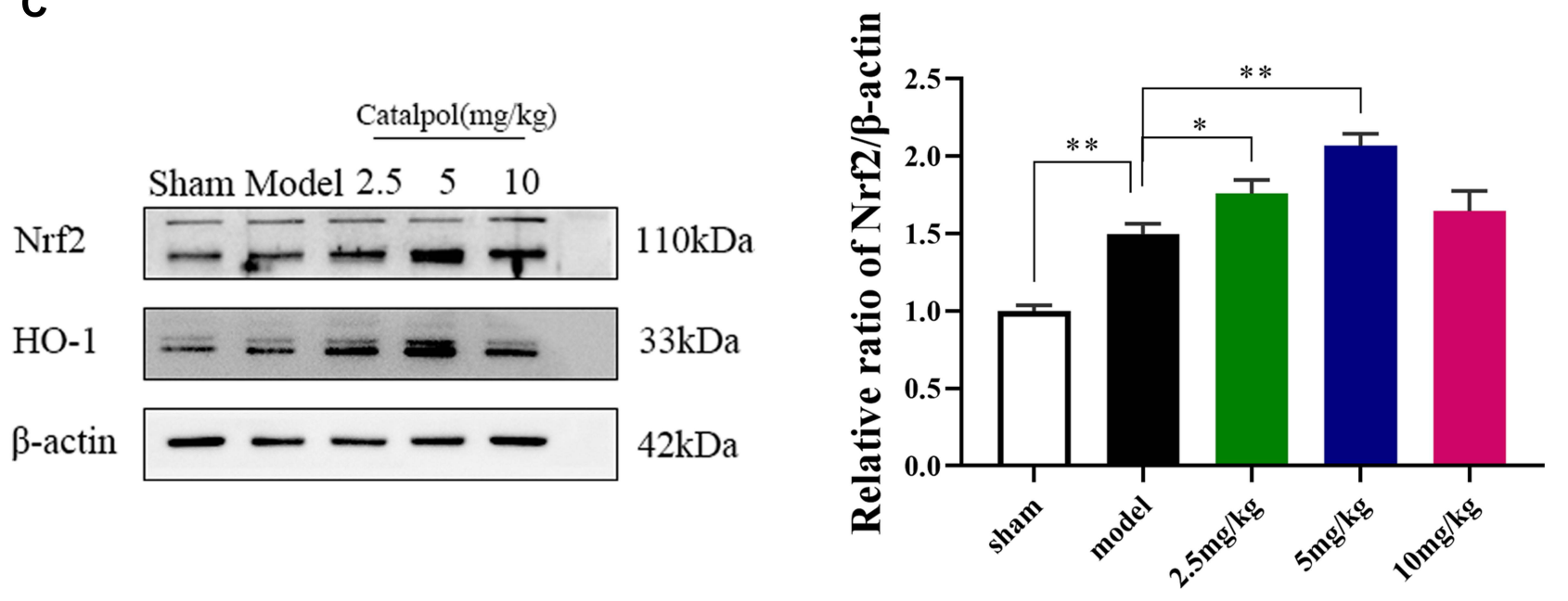

E

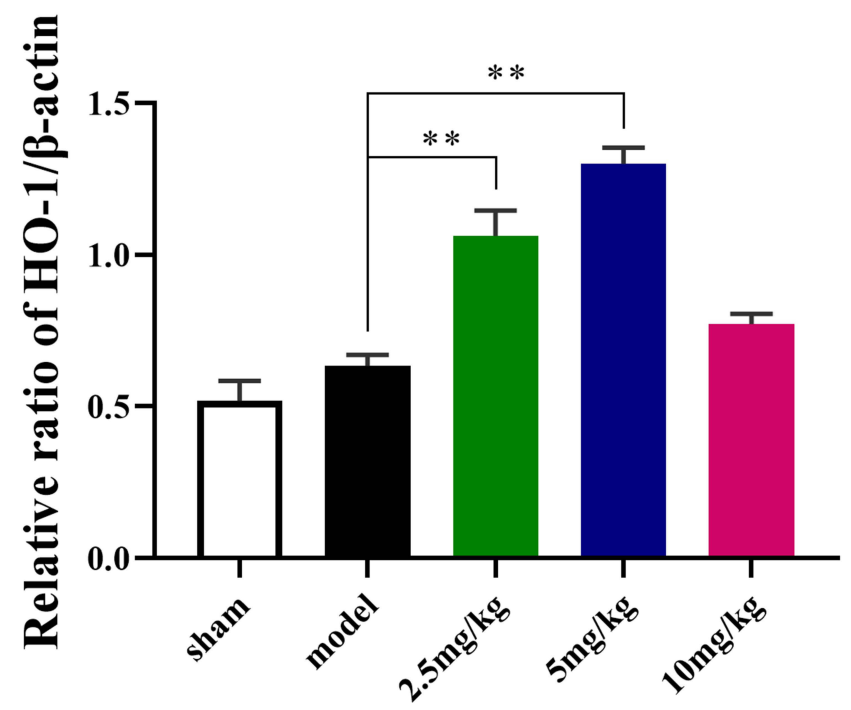

Figure 7 Effect of catalpol oxidative stress. (A) The content of MDA; (B) the content of SOD; (C) The activating effect of catalpol on the expression of Nrf2/HO-I pathway-related proteins; (D) the protein expression levels of $\mathrm{Nrf2}$; $(\mathbf{E})$ the protein expression levels of $\mathrm{HO}-\mathrm{I}$ (the data are presented as the mean $\pm \mathrm{S} . \mathrm{D}$., $\mathrm{n}=3$, $* \mathrm{p}<0.05$, $* * p<0.01)$. 

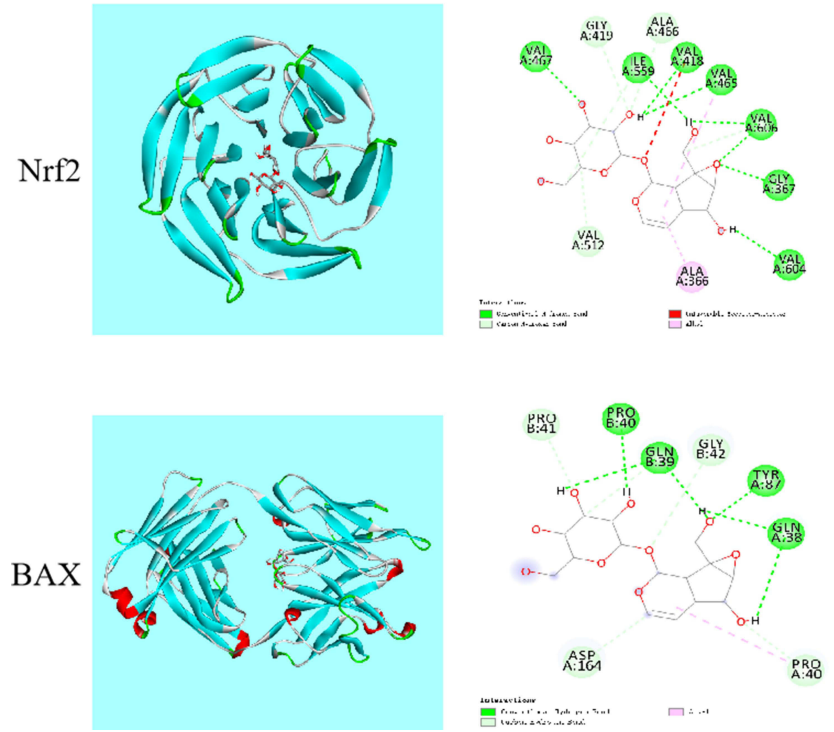

Figure 8 Graph of the small molecule and the target protein.

have a certain connection with the $\mathrm{CNS}^{27}$ It is because of the close connection between nose and brain nerve that nasal therapy is often used to treat acute clinical brain diseases. ${ }^{28}$ As a non-invasive way of drug administration, intranasal administration has attracted more and more attention. It can be used for local and systemic administration, especially in emergency treatment and long-term administration of chronic diseases. Compared with other conventional administration methods, it has the characteristics of rapid absorption, high bioavailability and direct access to the brain. ${ }^{29}$

In this study, we linked catalpol in the treatment of neurological damage caused by cerebral ischemia with intranasal administration, through hemolysis test and cilia toxicity test. The evaluation of the drug's toxic effect on nasal mucosa is mainly from three aspects: the effect on ciliary movement, the structural integrity of nasal mucosa and the release of transmitters. ${ }^{30}$ The commonly used evaluation methods: determining the effect of drugs on ciliary clearance, examining the morphology of nasal mucosa, hemolysis test and so on. ${ }^{31}$ We observed the hemolysis, ciliary toxicity and nasal mucosal toxicity of catalpol. Through the safety of the catalpol intranasal administration was assured by this study.

The BBB and the blood-cerebrospinal fluid barrier restrict the circulation of drugs from the system into the CNS. Although the BBB is used to protect the brain from various pathogens and toxic substances, it is also an important obstacle to the treatment of CNS disease. Due to the close relationship between nasal cavity and brain in anatomy and physiology, intranasal administration makes the drug avoid BBB and liver first pass metabolism, which is a practical, convenient and non-invasive way of drug administration. $^{32}$ In the way of drug transport in nasal mucosa, lipophilic and hydrophilic drugs and drugs with molecular weight less than 1000 can be absorbed through nasal mucosa. Catalpol has a molecular weight of 362.45 and strong hydrophilicity, so catalpol has the possibility of entering the nasal mucosa to further exert its efficacy. We analyzed the distribution of catalpol in plasma and brain tissue after intranasal administration of catalpol nasal drops, and discussed the brain targeting of catalpol intranasal administration.

After intranasal administration, the brain targeting index (DTI) of catalpol was much more than 1, indicating that catalpol intranasal administration had good brain targeting and increased the distribution of brain concentration. The accumulation of catalpol concentration in the brain could be achieved by nasal administration. The bioavailability of catalpol administered intranasally was higher than that in plasma. Catalpol intranasal administration is rapid absorption of brain-targeted drug delivery pathway. We speculate that catalpol can also achieve therapeutic effect in emergency.

In the pharmacodynamic study, we made use of the convenient and non-invasive characteristics of intranasal administration to observe the protective effect of catalpol after acute cerebral ischemia in rats. At present, the rat 
MCAO prepared by thread occlusion method is a recognized standard animal model in the study, and it has been widely used. Neurobehavioral score can indirectly evaluate the degree of brain damage after cerebral ischemia. We first used the mNSS score for neurological evaluation. ${ }^{33}$ In order to more accurately determine the degree of brain damage, we used the most direct detection index TTC staining. ${ }^{34}$ This result is also consistent with the neurological evaluation, indicating that MCAO could cause brain injury and then lead to neurological dysfunction. However, all dose groups of catalpol could significantly reduce the area of cerebral infarction. The results of HE staining (shown Figure 5) showed that in the MCAO group there were a large number of ischemic characteristics such as neuronal necrosis and edema in the cerebral cortex. It is further proved that cerebral ischemia can cause neuronal damage in the cerebral cortex. The results of $\mathrm{HE}$ staining showed that a large number of neuronal cells in the cerebral cortex of the MCAO group showed ischemic characteristics such as necrosis and obvious edema, which are consistent with the results reported in the literature. ${ }^{35}$ It further proves that cerebral ischemia can cause damage to cerebral cortex neurons. However, in the cerebral cortex of rats in each dose of catalpol, the number of neurons with ischemic characteristics was significantly reduced. These results further prove that catalpol has a protective effect on the brain.

At present, a large number of studies have shown that necrosis is the main form of death in the ischemic center, and Bcl-2-related proteins, which play an important role in the regulation of apoptosis, have become the focus of research. ${ }^{36}$ At present, 25 members of Bcl-2 family have been found in mammals, which can be divided into antiapoptotic Bcl-2 subfamily and pro-apoptotic Bax subfamily. ${ }^{37}$ Under physiological conditions, the antiapoptotic protein Bcl-2 and members of the pro-apoptotic protein Bax subfamily form a heterodimer, which prevents Bax from exerting its apoptotic effect. ${ }^{38}$ When stimulated by death signal, Bcl-2 protein is inactivated and Bax protein is released, which leads to cell apoptosis. In order to verify the occurrence of apoptosis in ischemic brain tissue, we used Western blot to detect the expression of apoptosis-related genes and proteins in each group. The results showed that in the model group, the expression of Bcl-2 protein with anti-apoptosis effect was significantly decreased, while the expression of Bax protein with apoptosis-promoting effect was significantly increased, which was consistent with the literature report. ${ }^{39}$ Catalpol can significantly promote the expression of Bcl-2 protein and inhibit the expression of Bax protein, indicating that catalpol can reduce the occurrence of apoptosis, and further verify the brain protective effect of catalpol.

It is well known that oxidative stress plays a fundamental role in cerebral ischemic injury. ${ }^{40}$ The assessment of oxidative stress is mainly realized by the following two parameters: level of MDA, which is used as an indicator of lipid peroxidation; and activity of SOD, which reflects antioxidant capacity. ${ }^{41}$ Our study showed that the activity of SOD in serum of the model group decreased significantly and the content of MDA increased significantly. The results showed that ischemic injury damaged the enzymatic antioxidant system, decreased the ability of scavenging free radicals, increased the production of free radicals, aggravated lipid peroxidation and caused brain damage. However, the content of MDA in catalpol each group was significantly decreased, and the activity of SOD was significantly increased, indicating that catalpol can scavenge free radicals by restoring the function of the antioxidant system and has an antioxidant effect. In addition to some small molecule enzymatic and non-enzymatic free radical scavengers, the body's antioxidant system also has the Nrf2 antioxidant signal pathway. The Nrf2 signaling pathway plays a key role in the antioxidant defense system against oxidative stress. $^{42}$ Expression of HO-1 is regulated by Nrf2. Oxidative stress encourages translocation of $\mathrm{Nrf} 2$ to the nucleus, where it upregulates the HO- $1 .^{43}$ So, in this study, the protein expression of transcriptional factors involved in the Nrf2/HO-1 redox signalling pathway including Nrf2 and HO-1 were explored. We further explored the role of Nrf2/HO-1 antioxidant signal pathway in cerebral ischemic injury. Western blot results showed that the expression of $\mathrm{Nrf} 2$ protein and $\mathrm{HO}-1$ protein in catalpol each group was significantly increased, indicating that catalpol may exert an antioxidant effect through the Nrf2/HO-1 signaling pathway. The results showed that oxidative stress reduced the scavenging of free radicals and aggravated lipid peroxidation by destroying the antioxidant system, which was involved in cerebral ischemic injury. Catalpol activates the Nrf2/HO-1 signaling pathway to exert an antioxidant effect and reduce brain damage.

Finally, we used molecular docking to verify the binding of catalpol and protein. The results showed that catalpol has certain binding effect with Nrf2, HO-1, Bcl-2, BAX, mainly through hydrogen bonding. 


\section{Conclusion}

In the present study, we demonstrated that catalpol intranasal administration has good safety, stability and brain targeting. It can effectively protect the brain injury of the rat model of acute cerebral ischemia and provide the possibility of drug administration in the acute stage of cerebral ischemia. The mechanism by catalpol intranasal administration exerts its protective effects involves activation of the oxidative stress pathway Nrf2/HO-1 and apoptosis pathway Bcl-2.

\section{Data Sharing Statement}

The data used to support the findings of this study are included within the article.

\section{Acknowledgments}

This work was supported by the NSFC Projects (81873034; 81073084), National Infrastructure of Chinese Genetic Resources[YCZYPT[2020]04; the Fundamental Research Funds for the Central Universities to the postgraduate (XDJK2017D154).

\section{Author Contributions}

All authors made a significant contribution to the work reported, whether that is in the conception, study design, execution, acquisition of data, analysis and interpretation, or in all these areas; took part in drafting, revising or critically reviewing the article; gave final approval of the version to be published; have agreed on the journal to which the article has been submitted; and agree to be accountable for all aspects of the work.

\section{Disclosure}

The authors declare that they have no conflicts of interest.

\section{References}

1. Stanford S, Sabra A, D'Silva L, et al. The changes in clot microstructure in patients with ischaemic stroke and the effects of therapeutic intervention: a prospective observational study. BMC Neurol. 2015;15:35. doi:10.1186/s12883-015-0289-1

2. Jian Z, Liu R, Zhu X, et al. The involvement and therapy target of immune cells after ischemic stroke. Front Immunol. 2019;10:2167. doi:10.3389/fimmu.2019.02167

3. Cabal M, Machova L, Vaclavik D, et al. New prehospital triage for stroke patients significantly reduces transport time of EVT patients without delaying IVT. Front Neurol. 2021;12:676126. doi:10.3389/ fneur.2021.676126

4. Ma W, Wang S, Liu X, et al. Protective effect of troxerutin and cerebroprotein hydrolysate injection on cerebral ischemia through inhibition of oxidative stress and promotion of angiogenesis in rats. Mol Med Rep. 2019;19(4):3148-3158. doi:10.3892/mmr.2019.9960
5. $\mathrm{Hu}$ J, Luo C, Chu W, et al. 20-Hydroxyecdysone protects against oxidative stress-induced neuronal injury by scavenging free radicals

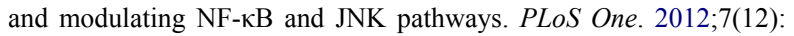
e50764. doi:10.1371/journal.pone.0050764

6. Li Y, Guan Y, Wang Y, Yu C, Zhai F, Guan L. $\gamma$ Neuroprotective effect of the ginsenoside $\mathrm{rg} 1$ on cerebral ischemic injury in vivo and in vitro is mediated by PPAR-regulated antioxidative and anti-inflammatory pathways. Evid Based Complement Alternat Med. 2017;2017: 7842082. doi:10.1155/2017/7842082

7. Xu J, Liu X, Luo L, Tang L, Guo N. A metabonomics investigation into the therapeutic effects of buchang naoxintong capsules on reversing the Amino acid-protein interaction network of cerebral ischemia. Oxid Med Cell Longev. 2019;2019:7258624. doi:10.1155/2019/7258624

8. Du F, Zhou L, Jiao Y, Bai S, Wang L, Ma J. Ingredients in Zijuan $\mathrm{Pu}$ 'er tea extract alleviate $\beta$-amyloid peptide toxicity in a Caenorhabditis elegans model of Alzheimer's disease likely through DAF-16. Molecules. 2019;24(4):729.

9. Chen J, He F, Liu S, Zhou T, Baloch S. Cytoprotective effect of ligustrum robustum polyphenol extract against hydrogen peroxide-induced oxidative stress via Nrf2 signaling pathway in Caco-2 cells. Evid Based Complement Alternat Med. 2019;20 19:5026458. doi:10.1155/2019/5026458

10. Shen B, Zhao C, Wang Y, et al. Aucubin inhibited lipid accumulation and oxidative stress via Nrf2/HO-1 and AMPK signalling pathways. J Cell Mol Med. 2019;23(6):4063-4075. doi:10.1111/jcmm.14293

11. Wang B, Han D, Li F. Elevated IL-22 in psoriasis plays an anti-apoptotic role in keratinocytes through mediating Bcl-xL/Bax. Apoptosis. 2020;25(9-10):663-673. doi:10.1007/s10495-020-01623-3

12. Wang $Z$, Huang $X$, Zhao $P$, Zhao L, Wang ZY. Catalpol inhibits amyloid- $\beta$ generation through promoting $\alpha$-cleavage of APP in Swedish mutant APP overexpressed N2a cells. Front Aging Neurosci. 2018;10:66. doi:10.3389/fnagi.2018.00066

13. Dong C, Liu S, Cheng X, Wang Q, Jiang S, Wang G. Design, synthesis, and preliminary biological evaluation of catalpol propionates as antiaging drugs. BMC Chem. 2019;13(1):109. doi:10.1186/ s13065-019-0626-3

14. Huang JZ, Wu J, Xiang S, et al. Catalpol preserves neural function and attenuates the pathology of Alzheimer's disease in mice. Mol Med Rep. 2016;13(1). doi:10.3892/mmr.2015.4496

15. Jiang Y, Zhang Q. Catalpol ameliorates doxorubicin-induced inflammation and oxidative stress in H9C2 cells through PPAR- $\gamma$ activation. Exp Ther Med. 2020;20(2):1003-1011. doi:10.3892/etm.2020.8743

16. Yang C, Shi Z, You L, Du Y, Ni J, Yan D. Neuroprotective effect of catalpol via anti-oxidative, anti-inflammatory, and anti-apoptotic mechanisms. Front Pharmacol. 2020;11:690. doi:10.3389/fphar. 2020.00690

17. Miles CO, Wilkins AL, Munday R. Catalpol inhibits apoptosis in hydrogen peroxide-induced PC12 cells by preventing cytochrome c release and inactivating of caspase cascade. Toxicon. 2004;43(1). doi:10.1016/j.toxicon.2003.10.003

18. Li D-Q, Duan Y-L, Bao Y-M, Liu C-P, Liu Y, An L-J. Neuroprotection of catalpol in transient global ischemia in gerbils. Neurosci Res. 2004;50(2). doi:10.1016/j.neures.2004.06.009

19. Li J, Yu J, Ma H, et al. Intranasal Pretreatment with Z-Ligustilide, the Main Volatile Component of Rhizoma Chuanxiong, Confers Prophylaxis against Cerebral Ischemia via Nrf2 and HSP70 Signaling Pathways. Journal of agricultural and food chemistry. 2017;65(8):1533-1542.

20. Yanlu Z, Michael C, Rex CS, et al. A small molecule spinogenic compound enhances functional outcome and dendritic spine plasticity in a rat model of traumatic brain injury. $J$ Neurotrauma. 2019;36 (4):589-600.

21. Dong W, Xian Y, Yuan W, et al. Catalpol stimulates VEGF production via the JAK2/STAT3 pathway to improve angiogenesis in rats' stroke model. J Ethnopharmacol. 2016;191:169-179. doi:10.1016/j. jep.2016.06.030 
22. Wang J, Wan D, Wan G, Wang J, Zhang J, Zhu H. Catalpol induces cell activity to promote axonal regeneration via the PI3K/AKT/ mTOR pathway in vivo and in vitro stroke model. Ann Transl Med. 2019;7(23):756. doi:10.21037/atm.2019.11.101

23. Cai M, Yu Z, Zhang W, et al. Sheng-Di-Da-Huang decoction inhibited inflammation expressed in microglia after intracerebral hemorrhage in rats. Evid Based Complement Alternat Med. 2018;20 18:1-10. doi:10.1155/2018/6470534

24. Zhu HF, Shao Y, Qin L. Catalpol enhances neurogenesis and inhibits apoptosis of new neurons via BDNF, but not the BDNF/Trkb pathway. Drug Design Dev Ther. 2019;13:4145-4157. doi:10.2147/ DDDT.S223322

25. Zhu H-F, Wan D, Luo Y, Zhou J-L, Chen L, Xu X-Y. Catalpol increases brain angiogenesis and up-regulates VEGF and EPO in the rat after permanent middle cerebral artery occlusion. Int J Biol Sci. 2010;6(5):443-453. doi:10.7150/ijbs.6.443

26. Illum L. Nasal drug delivery: new developments and strategies. Drug Discov Today. 2002;7(23):1184-1189. doi:10.1016/S1359-6446(02) 02529-1

27. Jalali S, Huang Y, Dumont DJ, Hynynen K. Focused ultrasound-mediated bbb disruption is associated with an increase in activation of AKT: experimental study in rats. BMC Neurol. 2010;10 (Suppl 1). doi:10.1186/1471-2377-10-114

28. Toshihiko T. Shortcut approaches to substance delivery into the brain based on intranasal administration using nanodelivery strategies for insulin. Molecules. 2020;25(21):5188.

29. Fatouh A, Elshafeey A, Abdelbary A. Intranasal agomelatine solid lipid nanoparticles to enhance brain delivery: formulation, optimization and in vivo pharmacokinetics. Drug Des Devel Ther. 2017;2017 (11):1815-1825.

30. Ugwoke MI, Agu RU, Verbeke N, Kinget R. Nasal mucoadhesive drug delivery: background, applications, trends and future perspectives. Adv Drug Deliv Rev. 2005;57(11):1640-1665. doi:10.1016/j.addr.2005.07.009

31. Vetter A, Augustijns P, Bernkop-Schnürch A. Solubilizing agents in nasal formulations and their effect on ciliary beat frequency. Toxicol in Vitro. 2012;26(1). doi:10.1016/j.tiv.2011.10.011

32. Haque S, Md S, Fazil M, et al. Venlafaxine loaded chitosan NPs for brain targeting: pharmacokinetic and pharmacodynamic evaluation. Carbohydr Polym. 2012;89(1):72-79. doi:10.1016/j.carbpol.2012. 02.051
33. He WM, Ying-Fu L, Wang H, Peng YP. Delayed treatment of $\alpha 5$ GABAA receptor inverse agonist improves functional recovery by enhancing neurogenesis after cerebral ischemia-reperfusion injury in rat MCAO model. Sci Rep. 2019;9(1):2287. doi:10.1038/s41598-019-38750-0

34. Bederson JB, Pitts LH, Germano SM, Nishimura MC, Davis RL, Bartkowski HM. Evaluation of 2, 3, 5-triphenyltetrazolium chloride as a stain for detection and quantification of experimental cerebral infarction in rats. Stroke. 1986;17(6). doi:10.1161/01.STR.17.6.1304

35. DongJu P, JuBin K, PhilOk K. Epigallocatechin gallate (EGCG) alleviates neuronal cell damage against focal cerebral ischemia in rats. J Vet Med Sci. 2020;82(5):639-645.

36. Ladokhin AS. Regulation of apoptosis by the Bcl-2 family of proteins: field on a brink. Cells. 2020;9(9):2121. doi:10.3390/cells9092121

37. Fabien L. Apoptosis and oncogenesis: give and take in the BCL-2 family. Curr Opin Genet Dev. 2011;21(1):12-20.

38. Peng X, Xiaoming Z, Yanfei L, Zhongyi M, Shuping S, Xinxue G. miR-9 inhibition of neuronal apoptosis and expression levels of apoptosis genes Bcl-2 and bax in depression model rats through notch pathway. Exp Ther Med. 2020;19(1):551-556.

39. Feng C, Wan H, Zhang Y, et al. Neuroprotective effect of danhong injection on cerebral ischemia-reperfusion injury in rats by activation of the PI3K-Akt pathway. Front Pharmacol. 2020;11:298. doi:10.3389/fphar.2020.00298

40. Zhao B, Yuan Q, Hou JB, Xia ZY. Inhibition of HDAC3 ameliorates cerebral ischemia reperfusion injury in diabetic mice in vivo and in vitro. J Diabetes Res. 2019;2019:8520856. doi:10.1155/2019/8520856

41. Wang X, Zhao Q, Shi H, et al. Oxidative stress is important in the pathogenesis of stress-related mucosal disease. Exp Ther Med. 2020;20(5):83. doi:10.3892/etm.2020.9211

42. Do NQ, Zheng S, Park B, et al. Camu-camu fruit extract inhibits oxidative stress and inflammatory responses by regulating NFAT and Nrf2 signaling pathways in high glucose-induced human keratinocytes.Molecules 2021;26(11):3174.

43. Alaofi AL. Sinapic acid ameliorates the progression of streptozotocin (STZ)-induced diabetic nephropathy in rats via NRF2/HO-1 mediated pathways. Front Pharmacol. 2020;11:1119. doi:10.3389/fphar.2020. 01119

\section{Publish your work in this journal}

Drug Design, Development and Therapy is an international, peerreviewed open-access journal that spans the spectrum of drug design and development through to clinical applications. Clinical outcomes, patient safety, and programs for the development and effective, safe, and sustained use of medicines are a feature of the journal, which has also been accepted for indexing on PubMed Central. The manuscript management system is completely online and includes a very quick and fair peer-review system, which is all easy to use. Visit http://www. dovepress.com/testimonials.php to read real quotes from published authors. 